\title{
Intrinsic alignments in redMaPPer clusters - I. Central galaxy alignments and angular segregation of satellites
}

\author{
Hung-Jin Huang, ${ }^{1 \star}$ Rachel Mandelbaum, ${ }^{1 \star}$ Peter E. Freeman, ${ }^{1,2}$ Yen-Chi Chen, ${ }^{1,2,3}$ \\ Eduardo Rozo, ${ }^{4}$ Eli Rykoff ${ }^{5}$ and Eric J. Baxter ${ }^{6}$ \\ ${ }^{1}$ McWilliams Center for Cosmology, Department of Physics, Carnegie Mellon University, Pittsburgh, PA 15213, USA \\ ${ }^{2}$ Department of Statistics, Carnegie Mellon University, Pittsburgh, PA 15213, USA \\ ${ }^{3}$ Department of Statistics, University of Washington, Seattle, WA 98195, USA \\ ${ }^{4}$ Department of Physics, University of Arizona, 1118 E. Fourth St, Tucson, AZ 85721, USA \\ ${ }^{5}$ SLAC National Accelerator Laboratory, Menlo Park, CA 94025, USA \\ ${ }^{6}$ Center for Particle Cosmology, Department of Physics, University of Pennsylvania, Philadelphia, PA 19104, USA
}

Accepted 2016 August 5. Received 2016 August 4; in original form 2016 May 2

\begin{abstract}
The shapes of cluster central galaxies are not randomly oriented, but rather exhibit coherent alignments with the shapes of their parent clusters as well as with the surrounding largescale structures. In this work, we aim to identify the galaxy and cluster quantities that most strongly predict the central galaxy alignment phenomenon among a large parameter space with a sample of 8237 clusters and 94817 members within $0.1<z<0.35$, based on the red-sequence Matched-filter Probabilistic Percolation cluster catalogue constructed from the Sloan Digital Sky Survey. We first quantify the alignment between the projected central galaxy shapes and the distribution of member satellites, to understand what central galaxy and cluster properties most strongly correlate with these alignments. Next, we investigate the angular segregation of satellites with respect to their central galaxy major axis directions, to identify the satellite properties that most strongly predict their angular segregation. We find that central galaxies are more aligned with their member galaxy distributions in clusters that are more elongated and have higher richness, and for central galaxies with larger physical size, higher luminosity and centring probability, and redder colour. Satellites with redder colour, higher luminosity, located closer to the central galaxy, and with smaller ellipticity show a stronger angular segregation towards their central galaxy major axes. Finally, we provide physical explanations for some of the identified correlations, and discuss the connection to theories of central galaxy alignments, the impact of primordial alignments with tidal fields, and the importance of anisotropic accretion.
\end{abstract}

Key words: galaxies: clusters: general-large-scale structure of Universe.

\section{INTRODUCTION}

In the framework of the standard cold dark matter (CDM)dominated Universe, cosmic structures grow hierarchically. Small galaxies form first, then merge and group together through channels of the filamentary network to form clusters of galaxies (White \& Rees 1978; Blumenthal et al. 1984). During the process of structure formation, the distribution and orientation of galaxies may be set by the surrounding gravitational tidal fields, or be disturbed by activities such as mergers or feedback processes due to supernova or active galactic nuclei. In this work, we refer to any net preferred

^E-mail: hungjinh @andrew.cmu.edu (H-JH); rmandelb@andrew.cmu.edu (RM) orientation towards some reference direction or any existing galaxy shape correlations caused by these physically induced events as intrinsic alignments (in contrast with the coherent alignments induced by gravitational lensing). For recent reviews, see Joachimi et al. (2015), Kiessling et al. (2015) and Kirk et al. (2015).

Intrinsic alignments occur on a variety of scales. On large scales, several Mpc and above, galaxies show a net tendency to align radially towards overdensities (e.g. Mandelbaum et al. 2006; Hirata et al. 2007; Okumura, Jing \& Li 2009; Joachimi et al. 2011), and more detailed analysis of the cosmic web indicates coherent alignments along the stretching direction of filaments (Chen et al. 2015b; Tempel et al. 2015; Rong, Liu \& Zhang 2016). One of the leading theoretical models for intrinsic alignments at large scales ( $\gtrsim 6 \mathrm{Mpc})$ is the linear alignment model, which relates the alignment strength 
linearly to the smoothed tidal field at the time of galaxy formation (Catelan, Kamionkowski \& Blandford 2001; Hirata \& Seljak 2004), or variations of that model that include non-linear evolution of the density field (Bridle \& King 2007; Blazek, Vlah \& Seljak 2015). Based on a sample of luminous red galaxies (LRGs), Singh, Mandelbaum \& More (2015) adopted the above alignment models to quantify the large-scale alignment amplitude as a function of several LRG properties. They found that the alignment amplitude becomes stronger towards more luminous LRGs residing in higher mass haloes (see also Hirata et al. 2007; Joachimi et al. 2011).

On small scales, within galaxy clusters, there are two types of alignments. The first type is the alignment of satellite major axes towards the centre of their host dark matter (DM) halo, for which the observational proxy usually is the brightest cluster galaxy (BCG). This is often called satellite (or radial) alignment. Satellite alignment is believed to originate from the tidal torque induced primarily from the gravitational field of the DM halo (Ciotti \& Dutta 1994; Kuhlen, Diemand \& Madau 2007; Faltenbacher et al. 2008; Pereira, Bryan \& Gill 2008; Tenneti et al. 2015a). Observationally, the existence of satellite alignment is still controversial, with Pereira \& Kuhn (2005), Agustsson \& Brainerd (2006), Faltenbacher et al. (2007), Singh et al. (2015) reporting detections of the signal, while Hung \& Ebeling (2012), Schneider et al. (2013), Chisari et al. (2014), Sifón et al. (2015) found no significant detection. Some of this tension may arise from selection effects, as discussed by Singh et al. (2015). In addition, Hao et al. (2011) cautioned about the possibility of spurious satellite alignment signals due to systematic errors (the contamination from the diffuse light from BCGs). We will report our measurement of satellite alignment in red-sequence Matched-filter Probabilistic Percolation (redMaPPer) clusters and present detailed systemic analysis in the upcoming Paper II. In this paper, we focus on the second type of alignment, called central galaxy alignment.

Central galaxy alignment refers to the tendency of the major axis of the central galaxy to align with that of its host DM halo, for which the observational signature is that satellites (which we use as a tracer of the DM halo shape) preferentially reside along the central's major axis direction. This type of alignment is also termed 'BCG alignment' in the literature, as it is often assumed that the brightest galaxy within each cluster is the central galaxy (the central galaxy paradigm; see van den Bosch et al. 2005). However, Skibba et al. (2011) showed that 40 per cent, and Hoshino et al. (2015) that 20-30 per cent, of BCGs are not the galaxies that are located closest to the centre of the cluster potential well. The fact that the redMaPPer algorithm identifies centrals not only based on their luminosity but also on their colour and local galaxy density enables us to select a more robust set of central galaxies for our intrinsic alignment study. Therefore, throughout this work, we will use the term 'central galaxy alignment' for our result, and keep the term 'BCG alignment' when referring to previous works that utilize the BCG as a proxy for the central galaxy.

Unlike satellite alignment, the observational evidence for central galaxy alignment is strong and uncontroversial (e.g. Sastry 1968; Binggeli 1982; Niederste-Ostholt et al. 2010), and it can be explained by two possible physical mechanisms. The first is the filamentary nature of matter accretion (Dubinski 1998), and the second is primordial alignment with the tidal field set by both the host DM halo and large-scale structure (Faltenbacher et al. 2008). Since central galaxy alignment is robustly detected with existing large data sets, many studies have investigated its dependence on physical predictors such as central galaxy luminosity, colour, host halo mass, redshift, and so on, in order to better understand the phys- ical origin of the effect (Brainerd 2005; Yang et al. 2006; Azzaro et al. 2007; Faltenbacher et al. 2007; Wang et al. 2008; Siverd, Ryden \& Gaudi 2009; Agustsson \& Brainerd 2010; NiedersteOstholt et al. 2010; Hao et al. 2011). There is general agreement that the central galaxy alignment signal is stronger for red and luminous centrals, and shows higher significance when using red satellites as tracers. However, some controversies still remain about the importance of other predictors besides luminosity or colour. Furthermore, some of the previous studies started with the assumption that only a few predictors could be important in determining the central galaxy alignments, and therefore performed an analysis based only on those predictors without considering others, ignoring potential degeneracies among predictors when splitting and comparing subsamples.

In Paper I, our goal is to present a comprehensive analysis of the predictors of central galaxy alignments. We include as many physical properties as possible, and properly account for potential correlations among them with the help of a linear regression analysis. We also discuss potential systematic effects based on signals obtained from various shape measurement methods. The two main questions we aim to address are (1) What central and cluster properties are the strongest predictors of the strength of central galaxy alignments? (2) What kinds of satellites are more likely to lie along the major axis direction of their host centrals? We build corresponding linear regression models, use variable selection techniques to select important predictors, and further quantify their significance. Finally, we discuss possible physical origins for these selected predictors and compare our result with the literature.

This paper is organized as follows. In Section 2, we describe our data and definitions of the physical quantities used in the linear regression analysis. Details of the linear regression process are described in Section 3. Section 4 presents our measurement of central galaxy alignment and results of the variable selection process. Section 5 discusses the detected central galaxy alignment signal for three different shape measurement methods, and the interpretation of those findings. The physical origins of our identified featured predictors for central galaxy alignments with the cluster shape and angular segregation of satellites with respect to the central galaxy major axis are discussed in detail in Sections 6 and 7, respectively. We conclude and summarize our key findings in Section 8 .

Throughout this paper, we adopt the standard flat $\Lambda \mathrm{CDM}$ cosmology with $\Omega_{\mathrm{m}}=0.3$ and $\Omega_{\Lambda}=0.7$. All the length and magnitude units are presented as if the Hubble constant were $100 \mathrm{~km} \mathrm{~s}^{-1} \mathrm{Mpc}^{-1}$. In addition, we use log as shorthand for the 10-based logarithm, and ln for the natural logarithm.

\section{DATA AND MEASUREMENTS}

In this section, we introduce the data that we analyse in this work, including the definitions of the galaxy cluster and galaxy properties that we use. All data used in this paper came from the Sloan Digital Sky Survey (SDSS) I/II surveys The SDSS I (York et al. 2000) and II surveys imaged roughly $\pi$ sr of the sky, and followed up approximately one million of the detected objects spectroscopically (Eisenstein et al. 2001; Richards et al. 2002; Strauss et al. 2002). The imaging was carried out by drift-scanning the sky in photometric conditions (Hogg et al. 2001; Ivezić et al. 2004), in five bands (ugriz; Fukugita et al. 1996; Smith et al. 2002) using a specially designed wide-field camera (Gunn et al. 1998). These imaging data were used to create the catalogues that we use in this paper. All of the data were processed by completely automated pipelines that detect and measure photometric properties of objects, and astrometrically calibrate the data (Lupton et al. 2001; Pier et al. 
2003; Tucker et al. 2006). The SDSS-I/II imaging surveys were completed with a seventh data release (Abazajian et al. 2009), but we use the processed data from an improved data reduction pipeline that was part of the eighth data release, from SDSS-III (Aihara et al. 2011); and an improved photometric calibration ('ubercalibration'; Padmanabhan et al. 2008).

\subsection{Galaxy cluster catalogue}

We use member galaxies in the redMaPPer v5.10 cluster catalogue ${ }^{1}$ to study galaxy alignments in galaxy clusters. The redMaPPer cluster catalogue is constructed based on photometric galaxy samples with a magnitude cut $m_{i}<21.0$ from the SDSS data release eight (DR8; Aihara et al. 2011) over a total area of $\sim 10000 \mathrm{deg}^{2}$. Details of the redMaPPer cluster finding algorithm and properties of the SDSS redMaPPer catalogues can be found in Rykoff et al. (2014), Rozo \& Rykoff (2014), Rozo et al. (2015a) and Rozo et al. (2015b). Briefly, the redMaPPer algorithm has two stages: the red-sequence calibration, and the cluster-finding stage. With a set of red spectroscopic galaxies as training sample, redMaPPer first constructs a redshift-dependent evolutionary red-sequence model, including zero-point, tilt and scatter. The calibrated red-sequence model is then used to group red galaxies at similar redshifts into clusters, assuming certain radial and luminosity filters.

One of the features of the redMaPPer algorithm is that it is probabilistic, which enables users to select suitable samples to do statistics. For each cluster, it provides the central galaxy probability, $P_{\text {cen }}$, for the top five potential BCGs, and all potential member galaxies are assigned with a membership probability, $p_{\text {mem }}$, according to their colour, magnitude and position information. The photometric redshift $z$ for each cluster is estimated from high-probability members; and the cluster richness, $\lambda$, is defined by summing the membership probabilities over all cluster members.

In this work, we restrict our analysis to clusters with richness $\lambda \geq 20$, corresponding to a halo mass threshold of $M_{200 \mathrm{~m}} \gtrsim$ $10^{14} h^{-1} \mathrm{M}_{\odot}$ (Rykoff et al. 2012), and photometric redshift in the range $0.1 \leq z \leq 0.35$. The lower redshift limit is selected so as to minimize edge effects from the training sample when doing calibration, while the upper redshift cutoff is set such that the sample of clusters is volume limited (Rykoff et al. 2014). All in all, there are 10702 clusters within this redshift and richness range. To perform higher quality statistics, we only explore satellite galaxies with membership probability $p_{\text {mem }} \geq 0.8$ when doing linear regression analysis, and restrict to satellites with $p_{\text {mem }} \geq 0.2$ when defining cluster shape (while weighting those satellites appropriately by their values of $\left.p_{\text {mem }}\right)$.

\subsection{Definitions and measurements of physical parameters}

In this subsection, we describe many of the physical parameters that we will use to study central galaxy alignments.

\subsubsection{Galaxy ellipticity}

The galaxy ellipticity used for the majority of this work is corrected for the effect of the point spread function (PSF) using the re-Gaussianization shape measurement method (see Section 2.3 for detail). We use the components of the distortion $e_{1}$ and $e_{2}$ (Bernstein \& Jarvis 2002) provided from the Reyes et al. (2012, or R12) and

\footnotetext{
${ }^{1}$ http://risa.stanford.edu/redmapper/
}

Mandelbaum et al. (2005, or M05) catalogues by fitting the 'atlas images' (Stoughton et al. 2002) in both $r$ and $i$ bands. The distortion can be related to the axis ratio $b / a$ as

$\left(e_{1}, e_{2}\right)=\frac{1-(b / a)^{2}}{1+(b / a)^{2}}(\cos 2 \alpha, \sin 2 \alpha)$,

where $\alpha$ is the position angle (PA) of the major axis. The total galaxy distortion $e$ is calculated as

$e=\sqrt{e_{1}^{2}+e_{2}^{2}}$.

\subsubsection{Galaxy alignment angles}

Once the galaxy PA is known, we can assign each satellite its central galaxy alignment angle, $\theta_{\text {cen }}$, and satellite alignment angle, $\phi_{\text {sat }}$.

The central galaxy alignment angle $\theta_{\text {cen }}$ is defined as the angle between the major axis of the central galaxy and the line connecting the central to the satellite galaxy, as illustrated in the left-hand panel of Fig. 1. Calculating $\theta_{\text {cen }}$ requires a viable shape measurement for the central galaxy (but not the satellites). Within the redshift range $0.1 \leq z \leq 0.35$, there are 8237 centrals with shape measurements in the R12 catalogue, resulting in 94817 central-satellite pairs with satellites that have $p_{\text {mem }} \geq 0.8$.

The satellite alignment angle $\phi_{\text {sat }}$ is defined as the angle between the major axis of the satellite galaxy and the line connecting its centre to the central, as shown in the right-hand panel of Fig. 1. Calculating $\phi_{\text {sat }}$ requires a shape measurement for the satellite galaxy. In this paper, we only consider $\phi_{\text {sat }}$ as a potential predictor of central galaxy alignments; future work will include a detailed analysis of satellite alignments.

We restrict both $\theta_{\text {cen }}$ and $\phi_{\text {sat }}$ to the range $\left[0^{\circ}, 90^{\circ}\right]$ due to symmetry. By definition, $\theta_{\text {cen }}=0^{\circ} / 90^{\circ}$ indicates a satellite located along the major/minor axis of the central. A satellite is radially/tangentially aligned with the central if $\phi_{\text {sat }}=0^{\circ} / 90^{\circ}$.

\subsubsection{Cluster PA and ellipticity}

We follow the method used in Niederste-Ostholt et al. (2010) to define the orientation and ellipticity of the redMaPPer clusters from their satellite distributions. In order to have enough member galaxies to trace the shape of each cluster, we use all member galaxies with membership probability $p_{\text {mem }} \geq 0.2$. We calculate the reduced second moments from the positions of member galaxies, weighted by $p_{\text {mem }}$ :

$M_{\mathrm{xx}} \equiv\left\langle\frac{x^{2}}{r^{2}}\right\rangle=\frac{\sum_{i} p_{\mathrm{mem}, i} \frac{x_{i}^{2}}{r_{i}^{2}}}{\sum_{i} p_{\mathrm{mem}, i}}$

and likewise for $M_{\mathrm{yy}}$ and $M_{\mathrm{xy}}$; by definition, $M_{\mathrm{xx}}+M_{\mathrm{yy}}=1$. Here $x_{i}$ is the distance of member galaxy $i$ from the cluster centre. We can then define the cluster ellipticity as

$(Q, U)=\frac{1-b / a}{1+b / a}(\cos 2 \beta, \sin 2 \beta)=\left(\mathrm{M}_{\mathrm{xx}}-\mathrm{M}_{\mathrm{yy}}, 2 \mathrm{M}_{\mathrm{xy}}\right)$,

where $b / a$ is the cluster projected minor-to-major axis ratio and $\beta$ is the cluster PA. The cluster ellipticity can then be calculated via

cluster $e=\sqrt{Q^{2}+U^{2}}$.

With the $1 / r^{2}$ weighting (an explicitly spherically symmetric weight function) in the reduced second moments, the derived cluster ellipticity tends to be underestimated. We show later that this does 

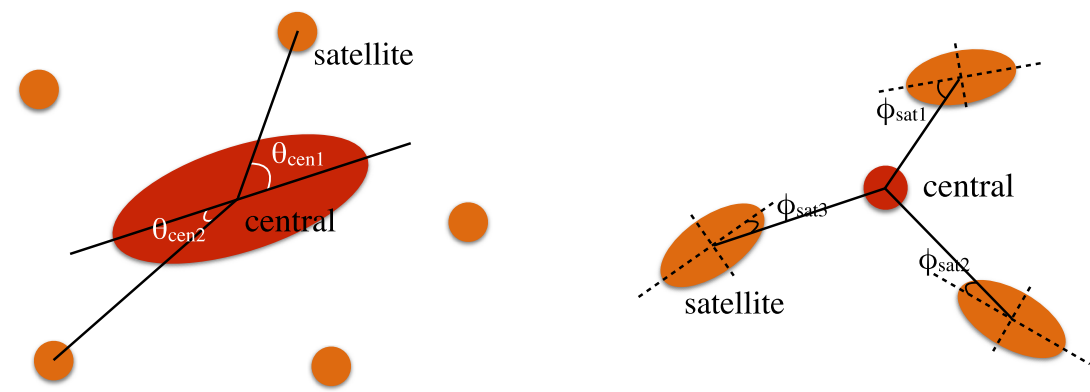

Figure 1. Illustration of the galaxy alignment angles. The left-hand panel shows the definition of central alignment angle $\theta_{\text {cen }}$, while the right-hand panel shows the definition of satellite alignment angle $\phi_{\text {sat }}$.

not change our conclusion regarding how cluster ellipticity affects the central galaxy alignment.

\subsubsection{Central galaxy dominance}

The central galaxy dominance parameter is defined as the difference in the $r$-band absolute magnitude of the central galaxy and the mean magnitude of the first and second brightest satellites:

Central dominance $\equiv$ Central $^{0.1} M_{\mathrm{r}}-\frac{{ }^{0.1} M_{\mathrm{r}, 1 \mathrm{st}}+{ }^{0.1} M_{\mathrm{r}, 2 \mathrm{nd}}}{2}$.

We calculate the central galaxy dominance parameter using only $p_{\text {mem }} \geq 0.8$ members. For the very few clusters (134 out of 8237) that have only one member satisfying the $p_{\text {mem }} \geq 0.8$ criterion, we simply use the difference between the absolute magnitudes of central and that member galaxy to define the central galaxy dominance. Smaller central dominance values correspond to more dominant central galaxies.

\subsubsection{Central galaxy probability}

For each cluster, the redMaPPer catalogue contains the five most likely central galaxy candidates, each with centring probability $P_{\text {cen }}$. In this paper, we use the most probable central as our central galaxy, and measure the central galaxy and satellite alignment angles of the associated central-satellite pairs. Over 80 per cent of our centrals have $P_{\text {cen }} \geq 0.7$.

\subsubsection{Galaxy absolute magnitude}

We calculate the absolute magnitude for each galaxy using the LRG templates in the KCORRECT package (v4.2) distributed by Blanton \& Roweis (2007). The KCORRECT software determines the best composite fit to the observed galaxy spectral energy distribution with the Chabrier (2003) initial mass function and a variety of Bruzual \& Charlot (2003) stellar population synthesis models differing in star formation histories and metallicities. We use extinction-corrected SDSS model magnitudes and the photometric redshift $z$ provided in redMaPPer as input, and $k$-correct the magnitudes of all galaxies in our sample to $z=0.1$.

\subsubsection{Galaxy effective radius}

The effective radius we report in this paper is the circularly averaged half-light radius, defined as

$R_{\mathrm{eff}} \equiv \sqrt{\frac{b}{a}} R_{\mathrm{deV}}$ where $b / a$ is the semiminor to semimajor axis ratio taken from the SDSS parameter deVAB_r, and $R_{\mathrm{deV}}$ is the semimajor half-light radius, deVRad_r. Both parameters are estimated as part of the SDSS DR8 pipeline by fitting de Vaucouleurs light profiles to galaxy $r$-band images. Here we convert the value of $R_{\mathrm{deV}}$ from the provided angular units to physical units $\left(h^{-1} \mathrm{kpc}\right)$, using the redshift $z$ of the host cluster.

\subsubsection{Member distance from the cluster centre}

For each satellite galaxy, we compute its projected distance, $r$, to the central galaxy, to check for radial dependence in the central galaxy alignment signal. To fairly compare among satellite galaxies in clusters with different halo masses, we further normalize $r$ by the estimated halo radius, $R_{200 \mathrm{~m}}$, corresponding to the radius within which the average density of the enclosed mass is 200 times the mean density, $\bar{\rho}$. We first use the mass-richness relation provided in equation B4 of Rykoff et al. (2012),

$\ln \left(\frac{M_{200 \mathrm{~m}}}{h_{70}^{-1} 10^{14} \mathrm{M}_{\odot}}\right)=1.72+1.08 \ln \frac{\lambda}{60}$,

to estimate $M_{200 \mathrm{~m}}$. Then we compute $R_{200 \mathrm{~m}}$ via the definition $M_{200 \mathrm{~m}}=(4 \pi / 3) 200 \bar{\rho} R_{200 \mathrm{~m}}^{3}$. Our conclusions would not change even if applying different mass-richness relations recently calibrated via weak lensing (Simet et al. 2016) or via clustering of clusters (Baxter et al. 2016).

\subsubsection{Cluster member concentration $\Delta_{R}$}

Recently, Miyatake et al. (2016) found that the average projected distance of member galaxies from the cluster centre, defined as

$\bar{R}_{\mathrm{mem}}=\frac{\sum_{i} p_{\mathrm{mem}, i} R_{i}}{\sum_{i} p_{\mathrm{mem}, i}}$,

not only describes the concentration of the member galaxy distribution in the cluster, but also plays a role in determining the large-scale clustering of redMaPPer clusters at fixed mass. Here $p_{\mathrm{mem}, i}$ is the membership probability of the $i$ th member galaxy, and $R_{i}$ is the physical separation between that galaxy and its corresponding cluster central galaxy.

To properly model the richness and redshift dependence in $\bar{R}_{\text {mem }}$, we use another parameter, $\Delta_{\mathrm{R}}$, defined in equation 22 of Baxter et al. (2016) as an indicator of cluster member concentration at fixed $\lambda$ and $z$ :

$\Delta_{\mathrm{R}}=\frac{\bar{R}_{\mathrm{mem}}-\left\langle\bar{R}_{\mathrm{mem}} \mid \lambda, z\right\rangle}{\left\langle\bar{R}_{\mathrm{mem}} \mid \lambda, z\right\rangle}$. 
Here $\left\langle\bar{R}_{\text {mem }} \mid \lambda, z\right\rangle$ is the mean $\bar{R}_{\text {mem }}$ value at a particular $\lambda$ and $z$ bin, estimated by fitting a spline to the average value of $\bar{R}_{\text {mem }}$ in 10 bins of $\lambda$ and five bins of $z$. By construction, negative $\Delta_{R}$ value means the cluster has a more compact member galaxy distribution than the average cluster at that richness and redshift.

\subsection{Galaxy shape data}

In this work, we use three different galaxy shape measurement methods from four catalogues to determine the galaxy PA and ellipticity, to investigate systematics in the measured central galaxy alignment signal. This section includes a description of all of these methods.

\subsubsection{Re-Gaussianization shape measurement}

The first shape measurement method is based on the reGaussianization technique (Hirata \& Seljak 2003), which not only corrects the effects of the PSF on the observed galaxy shapes with a standard elliptical Gaussian profile, but also corrects for loworder deviations from Gaussianity in both the galaxy and PSF profiles.

Two shape catalogues generated using the re-Gaussianization technique are used in this work; the primary one is based on the SDSS DR8 photometric pipeline, and was presented in R12; however, for systematics tests we also use the catalogue from M05, which was based on the DR4 photometric pipeline. The R12 catalogue covers an area of $9432 \mathrm{deg}^{2}$, with an average of 1.2 galaxies $\operatorname{arcmin}^{-2}$ with shape measurements; the M05 catalogue covers an area of $7002 \mathrm{deg}^{2}$. Both shape catalogues select galaxies down to the extinction-corrected $r$-band model magnitude $m_{r}<21.8$, and require galaxies to be well resolved compared to the PSF size in both $r$ and $i$ bands. While there are minor differences in galaxy selection criteria in the catalogues, the main difference is the version of the SDSS photometric pipeline (Рното) that they used. The M05 catalogue relies on Рното v5.4 (Adelman-McCarthy et al. 2006), while the R12 is based on Рното v5.6 (Aihara et al. 2011). The new version of Рното has a more sophisticated sky-subtraction algorithm that improves the photometry of large galaxies and fainter ones near them. By comparing the central galaxy alignment measured using these catalogues, we will estimate the impact of the sky-subtraction quality on the final results.

\subsubsection{Isophotal shape measurement}

Many previous central galaxy alignment studies used the SDSS isophotal PA to define the orientation of the BCG (Brainerd 2005; Yang et al. 2006; Azzaro et al. 2007; Faltenbacher et al. 2007; Wang et al. 2008; Siverd et al. 2009; Agustsson \& Brainerd 2010; Hao et al. 2011). To compare with these studies, we also measure the central galaxy alignment using the isophotal shape measurement. The SDSS pipeline measures the isophotal PA of galaxies at the isophote corresponding to $25 \mathrm{mag} \operatorname{arcsec}^{-2}$, which is fairly low surface brightness and generally encompasses a much larger part of the galaxy light profile than the centrally weighted re-Gaussianization shapes.

Isophotal shapes were not released in DR8, so we take the isophotal PA in $r$ band from DR7 (using the previous version of РНОто) to compute central galaxy alignments.

\subsubsection{De Vaucouleurs shape measurement}

Some galaxy alignment studies use the shape measurement from the de Vaucouleurs model fit (Siverd et al. 2009; Niederste-Ostholt et al. 2010; Hao et al. 2011), which is a good description of the surface brightness profile for a typical elliptical galaxy, including most galaxies in redMaPPer clusters. Here we use the de Vaucouleurs fit PA provided in the SDSS DR7, which fits galaxies through a two-dimensional fit to a PSF-convolved de Vaucouleurs profile. For more detail about these SDSS shape measurements, we refer readers to Stoughton et al. (2002).

\subsection{The central-satellite pair sample}

We define three samples of central-satellite pairs for our analysis.

(i) After applying the redshift cut and requiring that central galaxies have shape measurements in the R12 catalogue, we have 8237 centrals with DR8 re-Gaussianization shape measurement, and 94817 satellites with $p_{\text {mem }} \geq 0.8$ in our parent sample. This parent sample is used for the majority of our analysis, while the other subsamples are used primarily for systematics tests.

(ii) To investigate the effect of the sky-subtraction technique on the measured central galaxy alignment signal, we match our parent centrals with the M05 catalogue, and construct another subsample of centrals that have re-Gaussianization shape measurement based on both DR4 and DR8 photometry. This subsample has 4316 centrals and 46370 central-satellite pairs within the DR4 footprint.

(iii) To compare the degree of central galaxy alignment signal using different shape measurement methods, another subsample of central-satellite pairs is constructed. If we require centrals to have a DR8 re-Gaussianization shape, along with both isophotal and de Vaucouleurs shape measurements from DR7, we have 7488 centrals with 86350 satellites within the DR7 footprint.

Fig. 2 shows the distributions of the $r$-band absolute magnitude, ${ }^{0.1} M_{r}$, of the centrals (left-hand panel) and satellites (right-hand panel) in these three sets of central-satellite pairs. Both the central and satellite ${ }^{0.1} M_{r}$ distributions for the subsample in the DR7 footprint (iii) are almost the same as for the parent DR8 sample (i), while there are slight shifts for the subsamples in DR4 footprint (ii).

When measuring the central alignment angle, we only require satellite positions and central shape measurements. However, in the linear regression analysis, we require all galaxies to have welldefined physical parameters such as ellipticity, colour and effective radius; these requirements eliminate some satellites, mainly due to the requirement of an ellipticity measurement. Table 1 summarizes the three sets of central-satellite pairs defined in this section, and also records the actual number of central-satellite pairs used when doing linear regression analysis.

In Fig. 3, we compare the absolute magnitude distributions of the satellite subsamples actually used in the linear regression analysis to that of the original set of satellites from which they were drawn. The selected satellites used in linear regression are biased to brighter magnitudes, since we rely on good quality photometry (higher $\mathrm{S} / \mathrm{N}$ and/or more resolved light profile) to measure shapes. For the reason, the derived significance levels for potential predictors that could possibly affect the degree of central galaxy alignment in this work are lower limits, especially for predictors that strongly correlate with satellite brightness. If the effect of a predictor on central galaxy alignment is strong enough, then even if some faint satellites are excluded when doing linear regression, we could still select the predictor out as a featured predictor. 

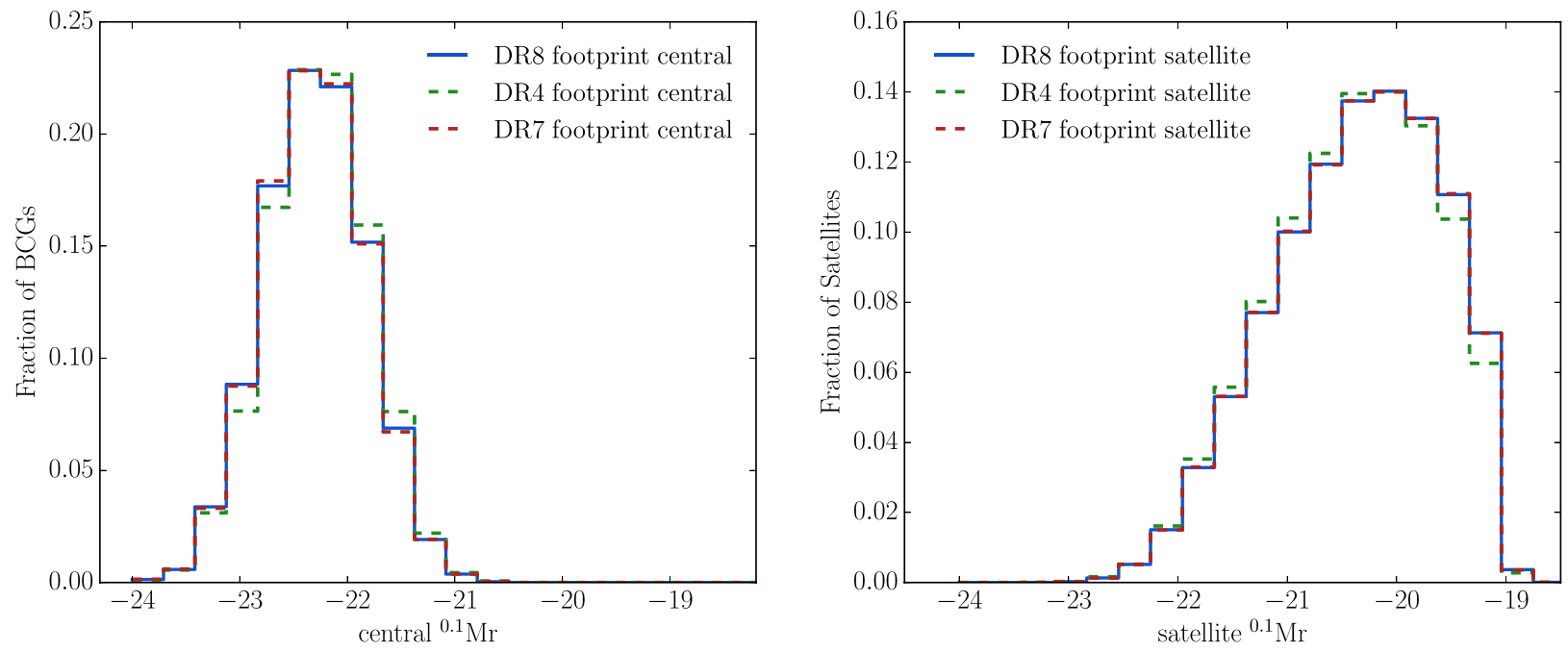

Figure 2. Distributions of the central (left-hand panel) and satellite (right-hand panel) ${ }^{0.1} M_{r}$ for the three sets of central-satellite pairs defined in Section 2.4 .

Table 1. Numbers of clusters and central-satellite pairs used in this work. The first three rows are the three subsamples we used for the overall measurement of the central galaxy alignment angle defined in Section 2.4. The last row is the subsamples used when doing linear regression analysis.

\begin{tabular}{lcc}
\hline Sample & $N_{\text {cluster }}$ & $N_{\text {pair }}$ \\
\hline DR8 footprint sample & 8237 & 94817 \\
DR4 footprint sample & 4316 & 46370 \\
DR7 footprint sample & 7488 & 86350 \\
Linear regression sample & 8233 & 73146 \\
\hline
\end{tabular}

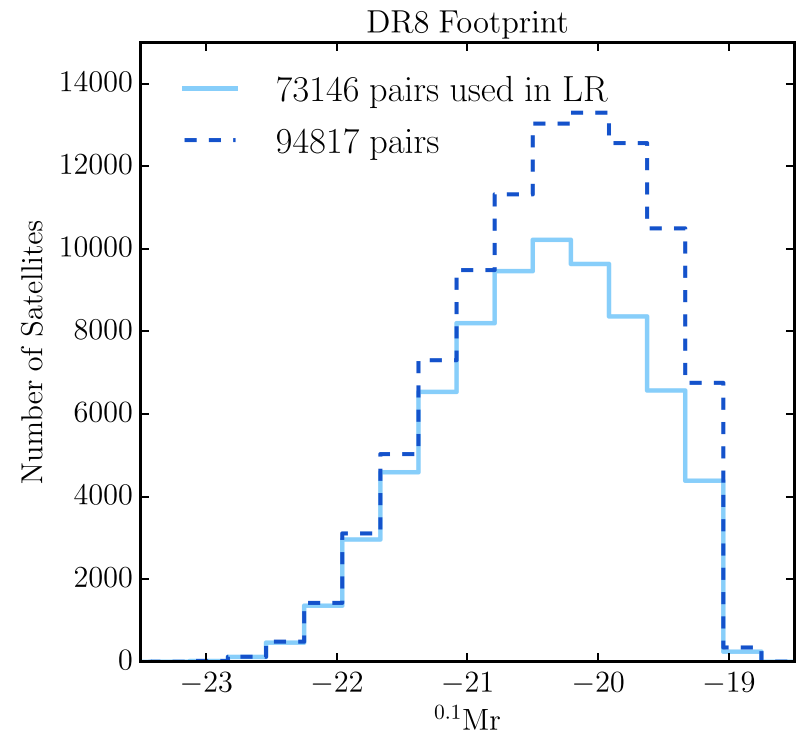

Figure 3. Distributions of the ${ }^{0.1} M_{r}$ for satellites in the DR8 footprint. The dark blue dashed line indicates the total 94817 satellites, while the light blue line shows the selected subsample when doing linear regression analysis.

\section{LINEAR REGRESSION ANALYSIS}

Regression is one of the most commonly used methods to study dependence. It is used to find optimal values of the free parameters in a specified function $Y=f(\boldsymbol{X})+\epsilon$. Here $Y$ is the response variable, which quantifies the physical effect one wants to study, $\boldsymbol{X}=\left(X_{1}, \ldots X_{i}, \ldots X_{N}\right)$ is a set of potential predictors that may affect the behaviour of $Y$, and $\epsilon$ represents random observational error, usually assumed to be drawn from a normal distribution. For the central galaxy alignment effect, as there is no a priori known functional form relating $\boldsymbol{X}$ to $Y$, we apply multiple linear regression, which allows one to at least determine if the central galaxy alignment depends on $\boldsymbol{X}$ to first order.

The multiple linear regression model we apply is

$Y=f(\boldsymbol{X})=\beta_{0}+\beta_{1} X_{1}+\cdots+\beta_{i} X_{i}+\cdots+\beta_{N} X_{N}$,

where the intercept $\beta_{0}$ and the slopes $\beta_{i}$ are the unknown regression coefficients to be estimated via least squares. For each regression coefficient $\beta_{i}$, we perform the two-sided $t$-value and $p$-value tests for the dependence of $Y$ on the $X_{i}$. These are tests of the hypothesis that $\beta_{i}=0$ against the alternative hypothesis that $\beta_{i} \neq 0$. The $t$ value is the ratio of $\beta_{i}$ to its standard error, which can be positive or negative depending on the sign of $\beta_{i}$. A larger $|t|$ indicates a more significant statement that $\beta_{i} \neq 0$, which means it is more likely that there is a relationship between $Y$ and $X_{i}$. Statistically, the $t$-value and $p$-value are inextricably linked. Under the assumption of normally distributed errors, a $p$-value of 0.05 corresponds to a 95 per cent confidence that $\beta_{i}$ is not equal to zero. Thus we select out a regressor $X_{i}$ as a featured predictor if its $p$-value $<0.05$ (e.g. Weisberg 2013).

There are reasons not to use our predictors $P_{i}$ defined in Section 2.2 (cluster ellipticity, central galaxy dominance, etc.) directly as regressors $X_{i}$. Since there is a large variation in the range of each predictor, if we simply regress by $Y=\beta_{0}+\sum_{i} \beta_{i}{ }^{\prime} P_{i}$, the fitted magnitude of $\beta_{i}^{\prime}$ would depend on that range, i.e. for a given level of correlation between the parameters, $\beta_{i}{ }^{\prime}$ would be small if its $P_{i}$ tends to be large. To make our results more directly illustrate how the relative change of a physical parameter affects the value of $Y$, 
Table 2. The 16 potential predictors used to study the central galaxy alignment effect in this work.

\begin{tabular}{lll}
\hline Central galaxy quantities & Cluster quantities & Satellite quantities \\
\hline Central galaxy dominance & $\log ($ richness $)$ & $\log \left(r / R_{200 \mathrm{~m}}\right)$ \\
Central ${ }^{0.1} M_{r}$ & Redshift & Satellite $M_{r}$ \\
Central $^{0.1} M_{g}-{ }^{0.1} M_{r}$ colour & Cluster ellipticity & Satellite ${ }^{0.1} M_{g}-{ }^{0.1} M_{r}$ colour \\
Central ellipticity & Cluster member concentration $\Delta_{\mathrm{R}}$ & Satellite ellipticity \\
$\Delta \log \left(\right.$ central $\left.R_{\text {eff }}\right)$ & & $\Delta \log \left(\right.$ satellite $\left.R_{\text {eff }}\right)$ \\
$P_{\text {cen }}$ & & $\phi_{\text {sat }}$ \\
\hline
\end{tabular}

throughout we normalize our predictors $P_{i}$ to obtain regressors as follows:

$X_{i}=\frac{P_{i}-\langle P\rangle_{i}}{\sigma_{P_{i}}}$.

Here $\sigma_{P_{i}}$ is the sample standard deviation of the predictor $P_{i}$, reflecting the width of the intrinsic distribution and measurement error. We will use the term 'predictor' to correspond to the original variables and 'regressor' to refer to variables that are transformed as in equation (12). We note, however, that using the normalized predictors as our regressors does not affect the result of hypothesis tests to select featured predictors.

In this work, we will build two multiple linear regression models with two different response variables, and use a total of 16 potential predictors to analyse the central galaxy alignment and the angular segregation of satellites. Details on the definitions and measurements of these physical parameters were presented in Section 2.2.

\subsection{Response variables}

The two response variables used to quantify the level of central alignment are: (1) the PA difference between the central galaxy and its host cluster

$\Delta \eta=\left|\mathrm{PA}_{\text {cen }}-\mathrm{PA}_{\text {cluster }}\right|$,

and (2) the central galaxy alignment angle for each central-satellite pair, $\theta_{\text {cen. }}$. We use these in different ways as described below.

$\Delta \eta$ lies in the range $\left[0^{\circ}, 90^{\circ}\right]$, where $0^{\circ}$ indicates that the central galaxy is perfectly aligned with the shape of the projected member galaxy distribution of the cluster. That distribution is believed to trace the underlying DM halo shape with some scatter (Evans \& Bridle 2009; Oguri et al. 2010). The quantity $\Delta \eta$ is thus an observable proxy for the level of central galaxy alignment with its DM halo. We will regress it on to central galaxy- and cluster-related predictors to identify what central galaxy properties and/or cluster properties most strongly predict the alignments of central galaxies with their satellite galaxy distributions.

The definition of $\theta_{\text {cen }}$ is illustrated in the left-hand panel of Fig. 1. It is a direct observable reflecting a satellite's angular position with respect to the major axis direction of its central galaxy. With each satellite galaxy having its corresponding $\theta_{\text {cen }}$ as the response variable, we will regress it on to individual satellite quantities to understand what kind of satellites are more preferentially located along the major axis of the central galaxy.

\subsection{Potential predictors}

We classify the 16 predictors into three categories: central-related, cluster-related and satellite-related quantities. Table 2 lists these predictors under each category.

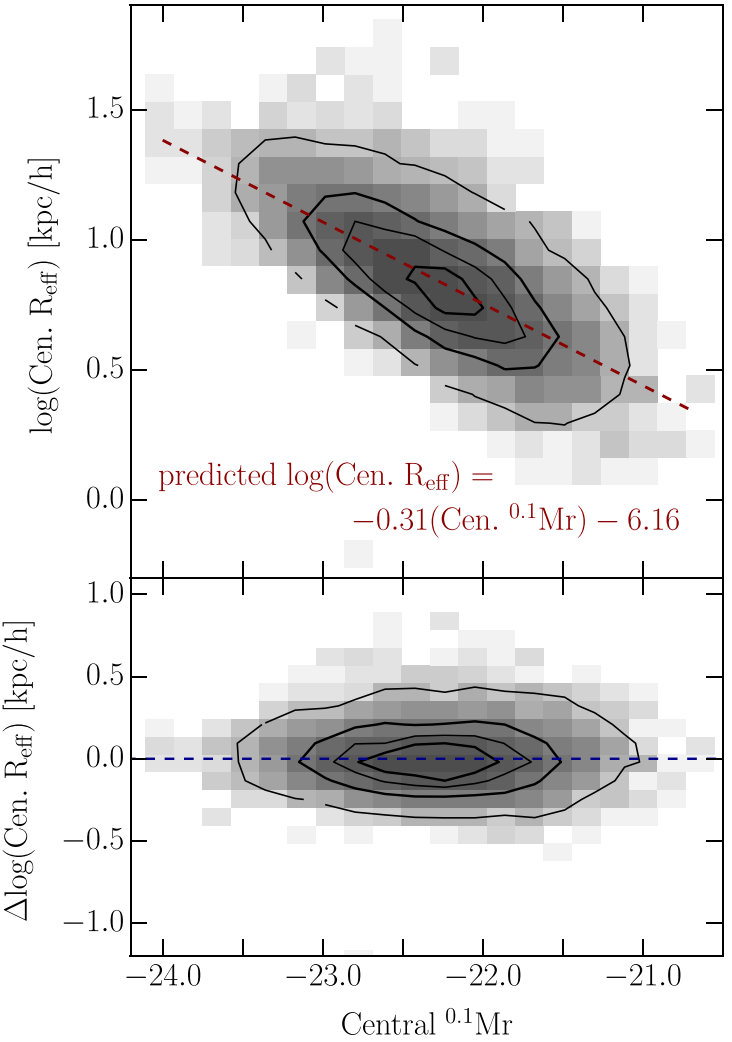

Figure 4. The top panel shows the contour plot of $\log$ (central $R_{\text {eff }}$ ) versus central ${ }^{0.1} M_{r}$. The red dash line shows the least-squares fitting of a linear relationship between $\log \left(\right.$ central $\left.R_{\text {eff }}\right)$ and central ${ }^{0.1} M_{r}$, with the equation of the best fitted line shown on the plot. The bottom panel plots the residuals versus central ${ }^{0.1} M_{r}$.

Central galaxy quantities. We use six central galaxy related physical parameters: central galaxy dominance, ${ }^{0.1} M_{r},{ }^{0.1} M_{g}-{ }^{0.1} M_{r}$ colour, ellipticity, effective radius and central probability. Since there is a tight correlation between the size and luminosity of galaxies (e.g. Bernardi et al. 2014), in order to investigate the effect of galaxy size on central galaxy alignments, we use the offsets in galaxy size from the fitted size-magnitude relation, $\Delta \log ($ central $\left.R_{\text {eff }}\right) \equiv$ measured $\log \left(\right.$ central $\left.R_{\text {eff }}\right)-$ predicted $\log \left(\right.$ central $\left.R_{\text {eff }}\right)$, as our predictor when doing linear regression. The top panel of Fig. 4 shows the $\log \left(\right.$ central $\left.R_{\text {eff }}\right)$-central ${ }^{0.1} M_{r}$ correlations for the DR8 central galaxies. In the bottom panel, we present the $\log \left(\right.$ central $\left.R_{\text {eff }}\right)$ residuals from the fitted $\log \left(\right.$ central $\left.R_{\text {eff }}\right)-$ central ${ }^{0.1} M_{r}$ relation, as a function of central ${ }^{0.1} M_{r}$.

Cluster quantities. We have four cluster-related physical parameters: $\log$ (richness), redshift, cluster ellipticity and cluster member concentration $\Delta_{\mathrm{R}}$. 


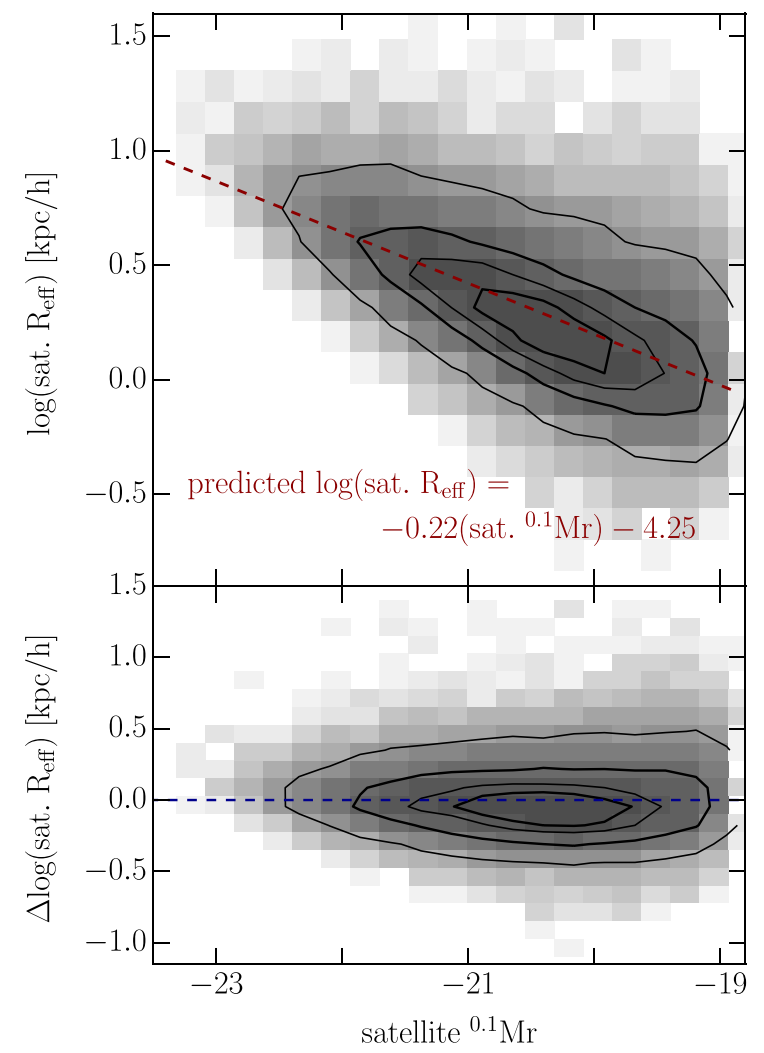

Figure 5. Similar to Fig. 4 but for cluster member galaxies instead of centrals.

Satellite quantities. The six satellite-related quantities are the cluster-centric distance of each satellite normalized by its host $R_{200 \mathrm{~m}},{ }^{0.1} M_{r},{ }^{0.1} M_{g}-{ }^{0.1} M_{r}$ colour, ellipticity, effective radius and the satellite alignment angle $\phi_{\text {sat }}$. As for the central galaxies, we use the residual effective radius, $\Delta \log \left(R_{\text {eff }}\right)$, for satellites as our physical parameter instead of the directly measured $R_{\text {eff }}$, in attempt to eliminate the contribution of luminosity on size. We fit the $\log \left(R_{\text {eff }}\right)-$ ${ }^{0.1} M_{r}$ correlations for the 73146 satellites as shown in the top panel of Fig. 5 first, and then use $\Delta \log \left(R_{\text {eff }}\right) \equiv$ measured $\log \left(R_{\text {eff }}\right)-$ predicted $\log \left(R_{\text {eff }}\right)$ as our new size predictor. The bottom panel of Fig. 5 shows the $\Delta \log \left(R_{\text {eff }}\right)$ as function of ${ }^{0.1} M_{r}$.

\subsection{Variable selection}

The goal of variable selection (e.g. Burnham \& Anderson 2003; James et al. 2013) is to identify the subset of predictors that are important within a large pool of potential predictors. There are many statistical methods for subset selection; we adopt the 'forwardstepwise selection' approach (see e.g. section 3.3 of Friedman, Hastie \& Tibshirani 2001). Beginning with a model containing no predictor, forward-stepwise selection involves fitting $N$ models for the $N$ predictors separately: $Y=\beta_{0}+\beta_{i} X_{i}$, and selects the regressor $X_{p}$ with the most significant hypothesis test on $\beta_{p} \neq 0$, i.e. greatest absolute $t$-value or smallest $p$-value. In the second cycle, $N-1$ models for the remaining $N-1$ predictors are fit via $Y=\beta_{0}+\beta_{p} X_{p}+\beta_{i} X_{i}$, where $i \neq p$, and again we select the most significant regressor $X_{q}$. At each stage, one predictor is selected to add to the model until the remaining regressors have $p$-value $>0.05$, which is a common stoping choice in many statistical pack- ages. The forward-stepwise algorithm therefore considers at most $N+(N-1)+\cdots+1=N(N+1) / 2$ models in the extreme case when all $N$ regressors have $p$-value $<0.05$. We then fit a model using least squares on the reduced set of variables, and determine the final $t$ - and $p$-values of the selected featured predictors.

To ensure the robustness of our variable selection scheme, we compare our variable selection result with another variable selection method - 'best-subset selection' - which considers all $2^{N}$ possible combinations of models from the $N$ predictors, and selects the best one based on a model-selection criterion, such as Mallow's $C_{p}$ (Mallows 1973), Akaike information criterion (AIC; Akaike 1998), Bayesian information criterion (BIC; Schwarz 1978), or adjusted $R^{2}$. (By contrast, forward-stepwise selection is a so-called greedy algorithm - at each step, it selects only that one regressor that best improves the overall fit - and thus it can fail to uncover the optimal model. However, it does have the virtue of computational efficiency.) Different section criterion places different penalty on the complexity of the model. BIC penalizes heavier on models with more variables and hence tends to select smaller number of predictors, while adjusted $R^{2}$ puts less penalty thus results in selecting more predictors. In this work, we use $p$-value $<0.05$ as the criteria to pin down the total number of predictors in the forward-stepwise selection process, and this result agrees with that from best-subset selection under Mallow's $C_{p}$ and AIC, validating our use of forwardstepwise selection with our data set.

Throughout this work, we use the statistical package STATSMODELS in PYTHON to do forward-stepwise selection, and use the LEAPS package in $\mathrm{R}$ to perform best-subset selection.

In this work, we attempt to address two main questions. (1) What central galaxy and cluster properties are the strongest predictors of the strength of central galaxy alignments? (2) What kinds of satellites are more likely to lie along the major axis direction of their host central galaxy? To address the first question, we regress $\Delta \eta$ against the central- and cluster-related quantities, and use forward-stepwise selection to pick featured predictors. Once we have a good model in terms of central- and cluster-related quantities, we move to the second question by using $\theta_{\text {cen }}$ as a response for each central-satellite pair, and regress $\theta_{\text {cen }}$ against the individual satellite quantities. To isolate the effects of satellite properties, we must properly account for the overall effect from their host central galaxies and clusters. Thus, we start with a model containing the selected central and cluster predictors from the previous stage, and use the forwardstepwise procedure to see whether (with the presence of these central and cluster quantities) there are also satellite quantities that are significant enough to be selected as featured predictors.

\section{RESULTS}

In this section, we report the results of an analysis of central galaxy alignments, including our linear regression analysis.

\subsection{Overall signal}

\subsubsection{Distribution of $\Delta \eta$}

We begin with a basic analysis of the properties of central galaxy alignments. Fig. 6 shows the distributions of the PA difference between the central galaxy and cluster shapes, $\Delta \eta$, for our three cluster samples tabulated in Table 1, defined for the purpose of investigating systemics in various shape measurement techniques. The distributions show a highly significant degree of central galaxy alignment with cluster orientations. The bottom-left corner of each 

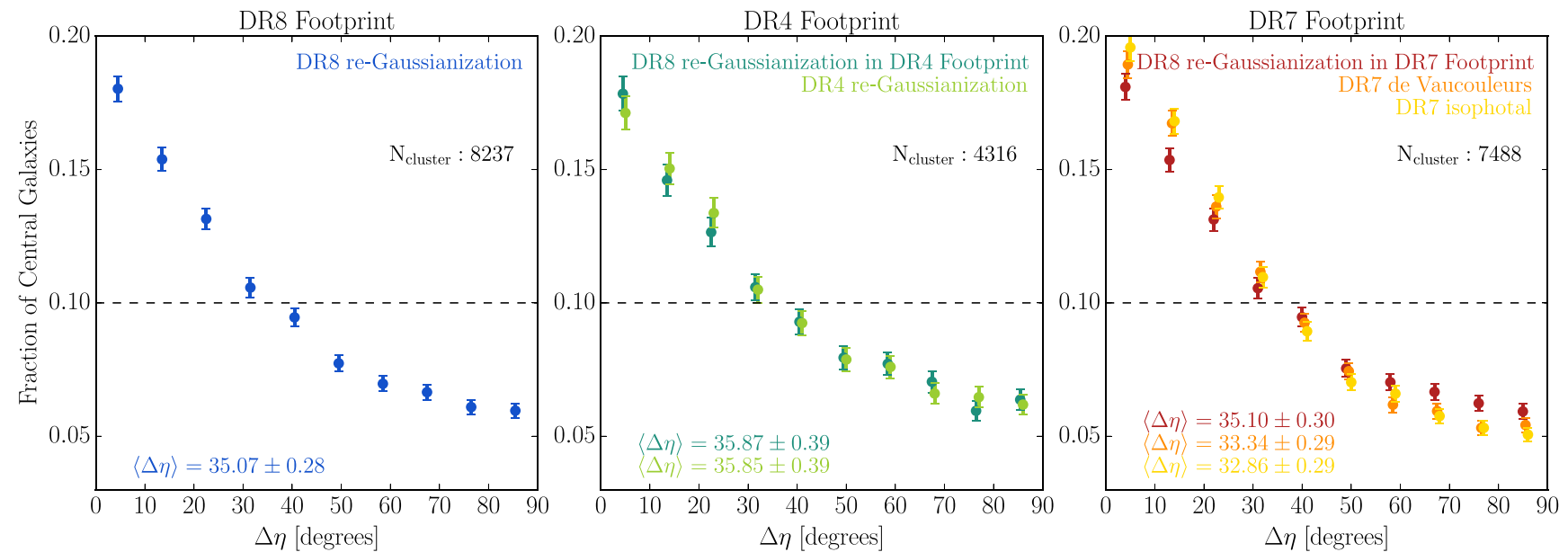

Figure 6. Distributions of the PA difference between central galaxy and cluster, $\Delta \eta=\left|\mathrm{PA}_{\mathrm{cen}}-\mathrm{PA}_{\text {cluster }}\right|$. The left-hand panel shows the $\Delta \eta$ distribution of the parent 8237 centrals measured by the re-Gaussianization method in DR8. The middle panel plots the $\Delta \eta$ distributions of the 4316 centrals which are both measured by the re-Gaussianization method in the DR4 footprint, with the light (dark) green dots representing measurements based on the DR4 (DR8) photometry. The right-hand panel shows the $\Delta \eta$ distributions of the 7488 centrals in DR7 footprint measured by re-Gaussianization (red dots), de Vaucouleurs (orange dots) and isophote (yellow dots) methods, respectively. Points are slightly shifted horizontally for clarity. Error bars indicate the standard error of the mean. The horizontal black dashed line indicates the prediction for randomly oriented central galaxies. The mean PA difference, $\langle\Delta \eta\rangle$, is shown in the bottom-left corner of each panel.

panel shows the average $\Delta \eta$ value, $\langle\Delta \eta\rangle$, for each sample; they are all $\langle\Delta \eta\rangle<45^{\circ}$ at high significance. Hence, if indeed the satellite galaxy distributions trace the DM halo shapes, then centrals also tend to align with their underlying haloes.

The left-hand panel of Fig. 6 is the $\Delta \eta$ distribution of the 8237 centrals measured by the re-Gaussianization method in DR8. The average $\Delta \eta$ for parent data set is $\langle\Delta \eta\rangle=35.07 \pm 0.28$; this represents our primary result in this section, with the remaining results serving as systematics tests.

To compare the effect of sky-subtraction algorithm on the signal, we use the sample of 4316 centrals in the DR4 footprint with re-Gaussianization shape measurements using both DR8 and DR4 photometry. The middle panel of Fig. 6 shows the $\Delta \eta$ distributions of this sample, with the light (dark) green dots indicating measurements based on the DR4 (DR8) photometry. Within the error bars, the two $\Delta \eta$ distributions and their mean $\langle\Delta \eta\rangle$ values are consistent with each other. We therefore conclude that for the re-Gaussianization shapes, the effect of sky-subtraction does not substantially influence the overall distribution of $\Delta \eta$. However, this conclusion may not be applicable for other shape measurement methods that trace different regions on the surface brightness profile of galaxies. Re-Gaussianization shapes are weighted more towards the inner part of the light profile, which is less sensitive to skysubtraction errors, while isophotal shapes are more sensitive to the outer part and could have more systematics due to sky subtraction. However, due to the lack of isophotal shapes in DR8, we cannot test this effect by comparing different data reductions.

To investigate the effect of shape measurement methods on the detection of central galaxy alignments, we use the sample of 7488 BCGs that have re-Gaussianization, de Vaucouleurs and isophotal shape measurements in DR7 footprint. The right-hand panel of Fig. 6 shows the $\Delta \eta$ distributions of these samples, with the red, orange and yellow dots representing shape measurements based on the re-Gaussianization method, de Vaucouleurs fit and isophotal fit, respectively. Within the error bars, the second and third distributions agree, while the $\Delta \eta$ distribution measured using the re-

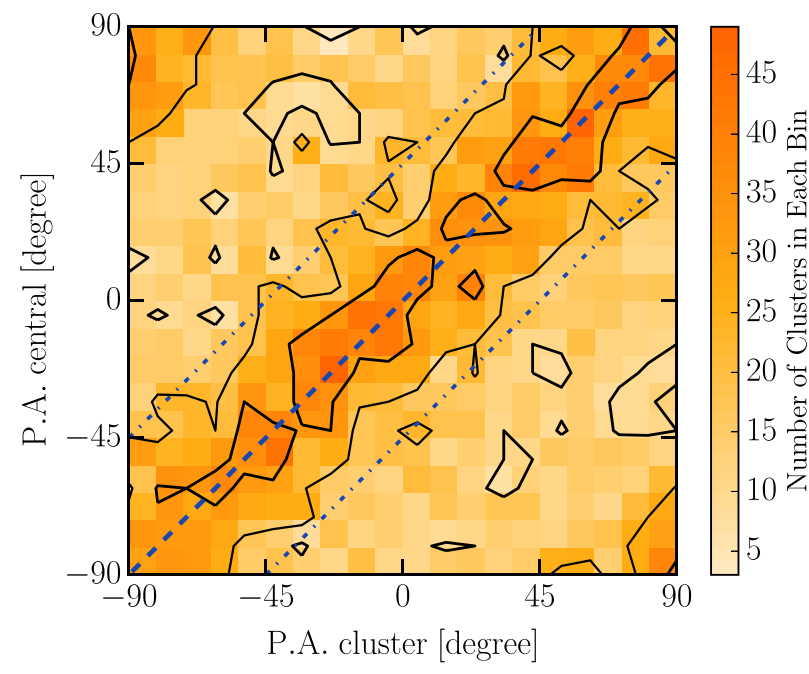

Figure 7. Comparison between the PAs of cluster and central galaxy. The blue dashed line indicates the case where the PA of the central is the same as that of its cluster. The two blue dot-dashed lines delineate a region where the PA differences between the cluster and central galaxy are less than $45^{\circ}$.

Gaussianization method differs systematically. The value of $\langle\Delta \eta\rangle$ using the re-Gaussianization method $\left(35^{\circ} .10 \pm 0.30\right)$ is significantly larger than that calculated by de Vaucouleurs (33.34 \pm 0.29$)$ and isophotal $(32.86 \pm 0.29)$ shape measurements. This could be due to a systematic or caused by a true physical effect. We will discuss in detail in Section 5.

As further illustration of the alignment between the central and shape of member galaxy distribution, in Fig. 7 we compare the cluster and central galaxy PAs. With the overall distribution peaking around the symmetric axis of the figure, we observe the preference for centrals pointing towards the orientation directions of clusters. 

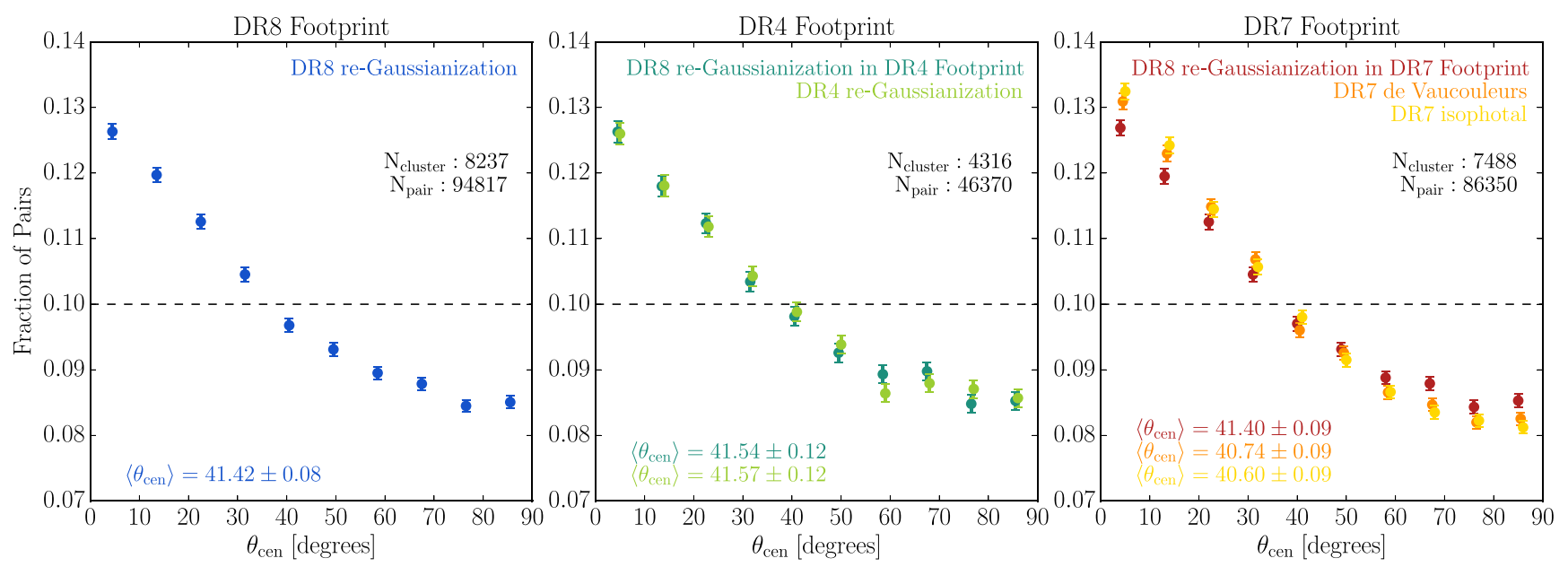

Figure 8. Distributions of the central galaxy alignment angle. The left-hand panel shows the $\theta_{\text {cen }}$ distribution of the 94817 central-satellite pairs measured by the re-Gaussianization method in DR8. The middle panel shows the $\theta_{\text {cen }}$ distributions of the 46370 central-satellite pairs measured by re-Gaussianization in both DR8 photometry (dark green) and DR4 photometry (light green), within the DR4 footprint. The right-hand panel shows the $\theta_{\text {cen }}$ distributions of the 86350 central-satellite pairs in DR7 that are measured by re-Gaussianization (red dots), de Vaucouleurs (orange dots) and isophote (yellow dots) methods, respectively. Points are slightly shifted horizontally for clarity. Error bars are represented by the standard error of the mean. The horizontal black dash line indicates the case if satellites were isotropically distributed around centrals. The mean central galaxy alignment angle, $\left\langle\theta_{\text {cen }}\right\rangle$, is shown at the bottom-left corner of each panel.

\subsubsection{Distribution of $\theta_{\text {cen }}$}

Fig. 8 shows the distributions of the central galaxy alignment angle, $\theta_{\text {cen }}$, for our three sets of central-satellite pairs. The preferential alignment of satellites along the central galaxy major axis is quantified in the average central galaxy alignment angle, $\left\langle\theta_{\text {cen }}\right\rangle$, in the bottom-left corner of each panel. The alignment signal looks less dramatic as revealed in $\left\langle\theta_{\text {cen }}\right\rangle$ value compared with $\langle\Delta \eta\rangle$ shown in Fig. 6. This is because $\theta_{\text {cen }}$ records the individual location of each satellite with respect to its central galaxy major axis; these tend to be more randomized than simply considering the overall satellite distribution as a whole.

The left-hand panel of Fig. 8 shows the $\theta_{\text {cen }}$ distribution of the 94817 central-satellite pairs measured by the re-Gaussianization method in DR8. The average central galaxy alignment angle for this data set is $\left\langle\theta_{\text {cen }}\right\rangle=41.42 \pm 0.08$.

The middle panel shows the $\theta_{\text {cen }}$ distributions for the 46370 central-satellite pairs in the DR4 footprint, using DR8 (dark green) and DR4 (light green) photometry, constructed to compare the effect of sky-subtraction technique on the measurement of $\theta_{\text {cen }}$. Within the error bars, the two $\theta_{\text {cen }}$ distributions and the derived $\left\langle\theta_{\text {cen }}\right\rangle$ values are consistent with each other. As for $\Delta \eta$, we conclude that for the reGaussianization shapes, use of different SDSS photometry pipelines does not influence the results, but caution that this argument may not hold for other shape measurement methods.

To fairly compare our $\theta_{\text {cen }}$ measurement with studies based on different shape measurement methods, the right-hand panel of Fig. 8 shows $\theta_{\text {cen }}$ measured via re-Gaussianization (red dots), de Vaucouleurs (orange dots) and isophote (yellow dots) shape measurements of the 86350 central-satellite pairs in the DR7 footprint. Within the error bars, the histograms of $\theta_{\text {cen }}$ using de Vaucouleurs and isophotal shapes are consistent with each other, but that for re-Gaussianization method is systematically different, resulting in a systematically higher $\left\langle\theta_{\text {cen }}\right\rangle$ $(41.40 \pm 0.09)$. We will discuss the measurement difference in Section 5 .

\subsection{Linear regression: central galaxy alignments with satellite distributions}

To investigate the alignment of the central galaxy with its DM halo, we use our observational proxy (the difference in central and cluster PAs, $\Delta \eta$ ) as the response variable, and apply forward-stepwise selection described in Section 3.3 to select featured predictors among the central- and cluster-related quantities. The list of predictors is defined in Section 3.2, with the observational method for determining them in Section 2.2.

Fig. 9 displays the scatterplot matrix between $\Delta \eta$ and all of the central- and cluster-related predictors based on the reGaussianization shape measurement. The diagonal panels are histograms of physical parameters, and the other panels are scatterplots between pairs of parameters, with the corresponding correlation coefficient noted on each plot.

Several important results are evident in this scatterplot matrix. First, the top row summarizes how $\Delta \eta$ is related to all 10 predictors. The sign of the correlation coefficient reveals the direction of the relationship between $\Delta \eta$ and the regressor, while the magnitude of the correlation coefficient indicates the strength of this dependence. The overall impression is that $\Delta \eta$ is weakly related to most of the predictors, with a maximum correlation coefficient of $\sim-0.2$ with cluster ellipticity. Though the correlations are weak, we can still judge whether these dependences are statistically significant given our large sample size. Secondly, some of the predictors are highly correlated with each other, such as central galaxy ${ }^{0.1} M_{r}$, central galaxy dominance and $P_{\text {cen }}$. The forward-stepwise selection procedure will help determine whether we should keep them all as featured predictors; if any are jointly responsible for the same variation in $\Delta \eta$, then we will select just the most representative one among them.

After performing forward-stepwise selection, we find that cluster ellipticity is the most dominant predictor for the central galaxy alignment effect, and almost all of the central-related quantities are selected as feature predictors except for central galaxy dominance. 


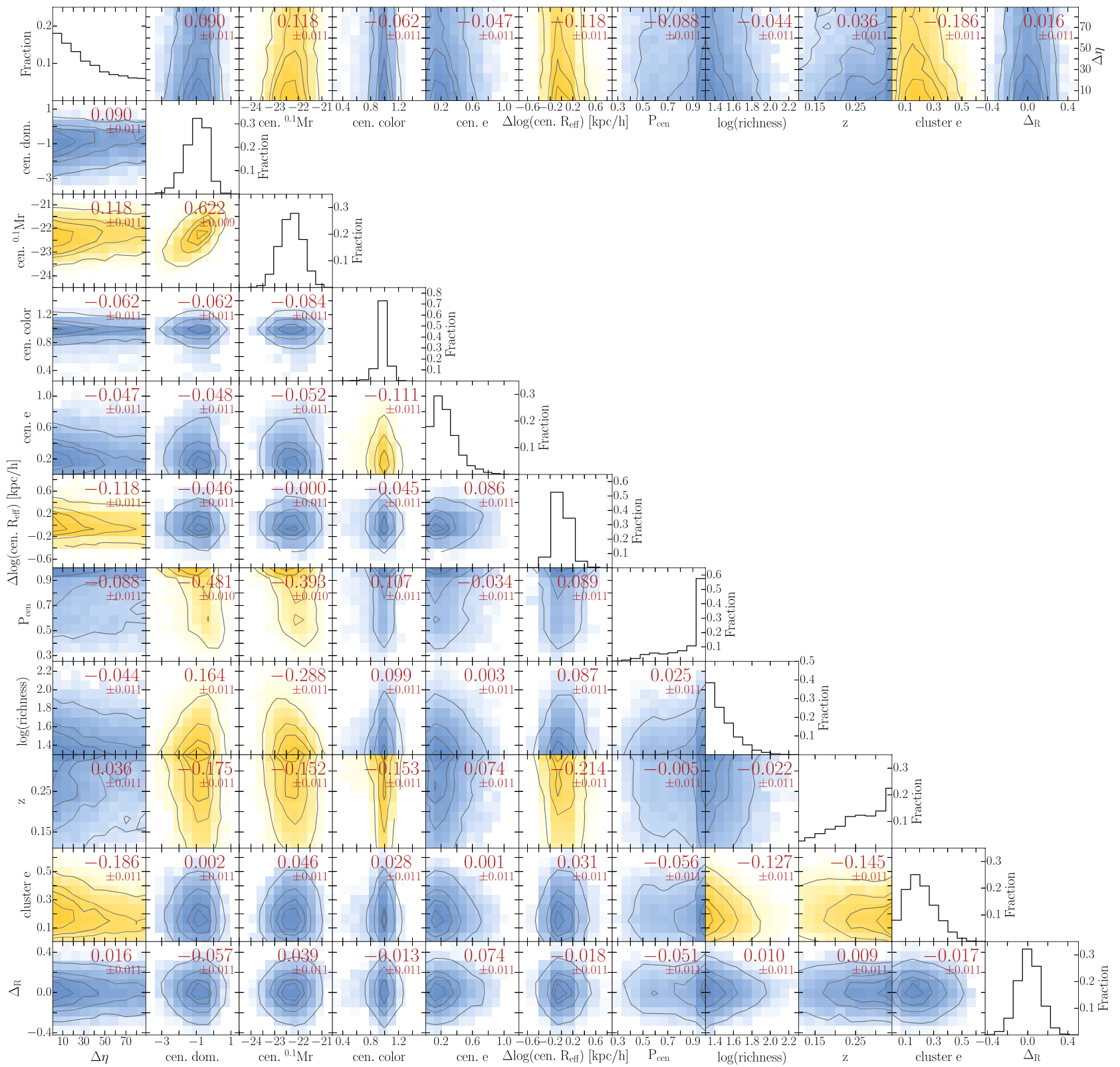

Figure 9. Scatterplot matrix of the PA difference between central and cluster shapes, $\Delta \eta$, with the 10 central- and cluster-related predictors. The correlation coefficient between each pair of parameters is noted on the plot. We highlight scatterplots with correlations that are significant at $>10 \sigma$ in yellow. The grey contour levels indicate 20, 40, 70 and 95 per cent number of clusters of our data.

The linear regression results, including the statistical significance of each selected predictor, are in Table 3, and the estimated best-fitting equation is

$$
\begin{aligned}
\Delta \eta= & 35.07-4.91 \frac{\text { cluster } e-0.21}{0.11} \\
& -2.67 \frac{\Delta \log \left(\text { cen. } R_{\text {eff }}\right)-0.00}{0.15}+2.42 \frac{\text { cen. }{ }^{0.1} M_{r}+22.30}{0.47} \\
& -1.29 \frac{\text { cen. colour }-0.97}{0.08}-1.21 \frac{P_{\text {cen }}-0.87}{0.17} \\
& -1.03 \frac{\text { cen. } e-0.26}{0.16}-0.65 \frac{\log (\text { richness })-1.48}{0.15}
\end{aligned}
$$

Here we note that since the relation between $\Delta \eta$ and these selected predictors is not truly linear and has substantial stochasticity, we cannot rely on the resulting regression equation to predict the value of $\Delta \eta$ for any given cluster. We can only use equation (14) to understand the sign and approximate strength of the variation of $\Delta \eta$ with those predictors to first order. Therefore, based on the trend of equation (14), we find that central galaxy alignment effects are strongest for clusters that are more elongated and higher richness, or clusters that have centrals with larger physical size, brighter absolute magnitude, redder colour, larger ellipticity ${ }^{2}$ and a higher centring probability.

\footnotetext{
${ }^{2}$ As we will demonstrate later in Section 6.5 , the dependence on central galaxy ellipticity is actually more complicated than this simple linear regression result indicates.
} 
Table 3. Selected featured predictors for the central galaxy-cluster alignment effect based on the 8233 DR8 clusters. The first column is the name of the selected predictor. The second column gives the regression coefficient $\beta$. Columns 3 and 4 provide the $t$ - and $p$-values from the significance tests on the deviation of $\beta$ from zero. Higher $|t|$ or smaller $p$ indicates a higher significance for $\beta \neq 0$. Columns 5 and 6 are the mean and standard deviation of the corresponding predictor for the 8233 clusters, necessary in equation (12) to normalize our predictor $P_{i}$ to regressor $X_{i}$.

\begin{tabular}{lrrlrr}
\hline Predictor & \multicolumn{1}{c}{$\beta$} & $t$-value & $p$-value & Mean & $\sigma$ \\
\hline Cluster $e$ & -4.91 & -17.8 & $8 \times 10^{-70}$ & 0.21 & 0.11 \\
$\Delta \log \left(\right.$ cen. $\left.\mathrm{R}_{\text {eff }}\right)$ & -2.67 & -9.7 & $6 \times 10^{-22}$ & 0.00 & 0.15 \\
cen. ${ }^{0.1} M_{r}$ & 2.42 & 7.8 & $9 \times 10^{-15}$ & -22.30 & 0.47 \\
cen. colour & -1.29 & -4.6 & $4 \times 10^{-5}$ & 0.97 & 0.08 \\
$P_{\text {cen }}$ & -1.21 & -4.0 & $6 \times 10^{-5}$ & 0.87 & 0.17 \\
cen. $e^{a}$ & -1.03 & -3.7 & 0.0002 & 0.26 & 0.16 \\
$\log$ (richness) & -0.65 & -2.2 & 0.03 & 1.48 & 0.15
\end{tabular}

$\overline{{ }^{a}}$ The relationship between central galaxy ellipticity and the central galaxy alignment signal is more complicated. We will provide further investigation in Section 6.5.

\subsection{Linear regression: angular segregation of satellite galaxies}

In Section 4.2, we used the positions of satellite galaxies weighted by their membership probabilities to trace the cluster and underlying halo shape, without any consideration of individual satellite properties. However, satellite galaxies with different properties are known to be distributed in different ways within clusters, a phenomenon known as segregation (e.g. van den Bosch et al. 2016). Segregation is often discussed in terms of the radial direction to the cluster centre. Here we investigate angular segregation with respect to the central galaxy major axis, to understand what satellite properties most strongly predict the satellite tendency to lie along the central galaxy major axis.

Fig. 10 shows the scatterplot matrix of $\theta_{\text {cen }}$ versus the six satelliterelated quantities for the 73146 DR8 central-satellite pairs. The top row displays scatterplots between $\theta_{\text {cen }}$ and all other satellite quantities. Compared with Fig. 9, the absolute magnitudes of the correlation coefficients of $\theta_{\text {cen }}$ with these satellite quantities are generally smaller than the correlation between $\Delta \eta$ and central and cluster quantities. Although the correlations are weak, the large number of pairs means there is still enough statistical power to measure these correlations robustly. In general, the relationships between all pairs of predictors appear to be weak, except for ${ }^{0.1} M_{r}$ and ellipticity, with a correlation coefficient of 0.236 . The lowerright corner shows the distribution of $\phi_{\text {sat }}$, which is very close to flat, indicating that satellite radial alignment is a far weaker phenomenon compared to central galaxy alignments; we explore this phenomenon in more detail in future work.

Given that we already identified the important central galaxy- and cluster-related predictors that affect the central galaxy alignment signal, it is reasonable to include these predictors in our linear regression analysis in order to compensate for their influence on the angular segregation of satellites. Table 4 shows the results of linear regression, with the new selected satellite quantities on top and the already known central galaxy and cluster quantities on the bottom. Almost all previously selected quantities have an associated $p$-value below 0.05 when using $\theta_{\text {cen }}$ as the response variable, except for cluster richness $\lambda$. This may be due to the fact that higher richness clusters tend to be rounder (as revealed in the last row of Fig. 9), and thus have their member galaxies less segregated towards any specific direction. Also, although the regression slope for $\log$ (richness) is positive, we cannot infer that satellites in lower richness clusters tend to be more segregated (i.e. having smaller $\theta_{\text {cen }}$ ). The level of angular segregation against richness is not significant enough for us to make such a conclusion. The best-fitted linear regression equation with these predictors is

$$
\begin{aligned}
\theta_{\text {cen }}= & 41.60-0.66 \frac{\text { sat. colour }-0.91}{0.09}+0.54 \frac{\text { sat. }{ }^{0.1} M_{r}+20.46}{0.76} \\
& +0.21 \frac{\log \left(r / R_{200 \mathrm{~m}}\right)+0.87}{0.32}+0.20 \frac{\text { sat. } e-0.43}{0.26} \\
& -2.52 \frac{\text { cluster } e-0.21}{0.11}-0.64 \frac{\Delta \log \left(\text { cen } R_{\text {eff }}\right)-0.02}{0.15} \\
& +0.58 \frac{\operatorname{cen}^{0.1} M_{r}+22.35}{0.49}-0.28 \frac{\text { cen colour }-0.98}{0.07} \\
& -0.46 \frac{P_{\text {cen }}-0.87}{0.17}-0.42 \frac{\text { cen } e-0.25}{0.16} \\
& +0.09 \frac{\log (\text { richness })-1.58}{0.21} .
\end{aligned}
$$

The tendency of satellites to reside along the central galaxy major axis is strongest for satellites that are redder, brighter, rounder and located closer to the central.

\section{THE EFFECT OF SHAPE MEASUREMENT METHOD ON THE CENTRAL GALAXY ALIGNMENT SIGNAL}

In the rightmost panels of Figs 6 and 8, we compared the distributions of our response variables, $\Delta \eta$ and $\theta_{\mathrm{BCG}}$, using three different shape measurements methods. In both cases, the level of central galaxy alignment measured via de Vaucouleurs and isophotal shapes agree with each other within the error bar, while the reGaussianization measurement gives us a less strong central galaxy alignment effect. In this section, we discuss the interpretation of this result in terms of systematic and physical effects in these shape measurements. Our discussion also relies on results of Singh \& Mandelbaum (2016), who analysed the effect of these three shape measurement methods on the inferred galaxy alignments of LRGs.

\subsection{Systematic error}

Different shape measurements deal with the effects of the PSF on galaxy images differently. The re-Gaussianization technique was designed for weak lensing studies requiring the most complete removal of the PSF effect on galaxy shapes. The de Vaucouleurs shape measurement only partially corrects for the PSF by using a doubleGaussian fit instead of the full PSF model, while the isophotal shape measurement does not correct for the effect of the PSF explicitly.

Another relevant aspect of systematics has to do with what part of the light profile is used for the measurement. The re-Gaussianization method has an elliptical Gaussian weight function, emphasizing the central regions of the profile. The de Vaucouleurs profile includes both the central region and the large-scale wings of the light profile, while the isophotal shape measurement only uses the $25 \mathrm{mag} \operatorname{arcsec}^{-2}$ isophote which is quite far out in the wings. These choices could make the latter two methods more sensitive to skysubtraction systematics than the re-Gaussianization method (for more discussion in the context of the isophotal method, see Hao et al. 2011).

One could infer that isophotal shapes would contain severe systematics due to the PSF. However, the results of Singh \& 


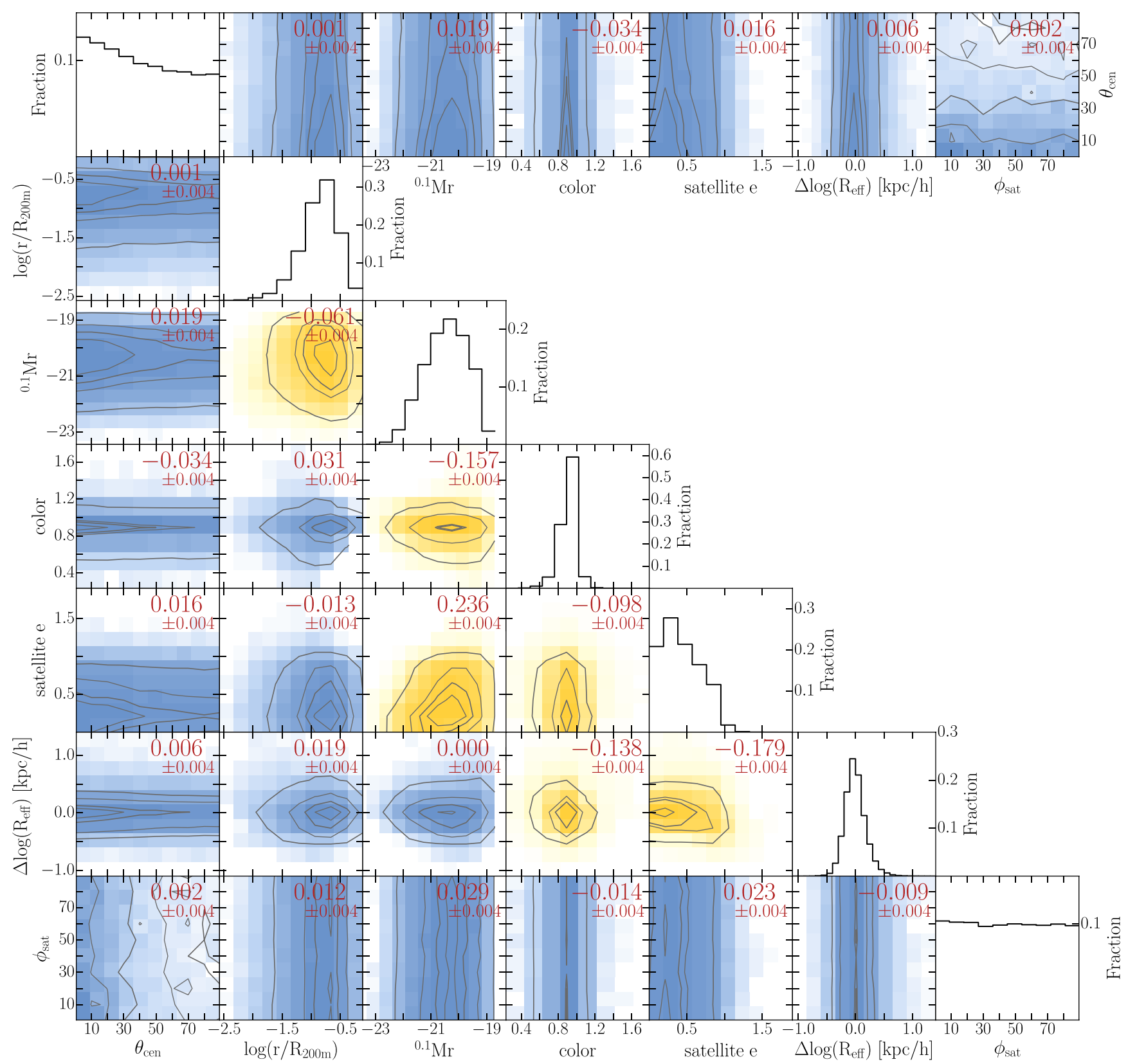

Figure 10. Scatterplot matrix of the central galaxy alignment angle with the six satellite related quantities. The correlation coefficient between each pair of parameters is noted on the plot. We highlight scatterplots with correlations that are significant at $>10 \sigma$ in yellow. The grey contour levels indicate 20 , 40 , 70 and 95 per cent number of satellites of our data.

Mandelbaum (2016) suggest that the impact of the PSF on the shape measured at very low surface brightness is quite small. Instead, the de Vaucouleurs shapes exhibited the most significant systematic errors of the three methods. Therefore, we may treat the detected differences in the central galaxy alignment strength between the reGaussianization and isophotal shapes as reflecting a true physical effect that we will discuss below. However, we should keep in mind that the systematic tests in Singh \& Mandelbaum (2016) were based on a specific sample of galaxies, while our central galaxy sample (which is preferentially located in regions of high galaxy density) may still suffer from some contamination in the isophotal shapes, as suggested by Hao et al. (2011).

\subsection{Physical effect}

The higher apparent degree of central galaxy alignment using isophotal shapes compared to that using re-Gaussianization shapes may be primarily due to a mechanism called 'isophote twisting' (di Tullio 1978, 1979; Kormendy 1982; Romanowsky \& Kochanek 1998; Lauer et al. 2005). The physical origin of this effect is that the outer part of the galaxy light profile may respond more strongly to tidal fields than the inner part of the galaxy. Thus, by tracing the outermost isophote of the galaxy, the isophotal shape records the highest level of alignment with the tidal field. 
Table 4. Predictors involved in the angular segregation of satellites as analysed in Section 4.3. The columns are the same as in Table 3. The bottom panel lists the already identified central galaxy and cluster quantities. These quantities are included in the linear regression equation when doing variable selection, in order to properly account for their influence on the angular segregation of satellites. The top panel shows the four selected satellite quantities that significantly affect angular segregation.

\begin{tabular}{lcclrr}
\hline Predictor & $\beta$ & $t$-value & $p$-value & Mean & $\sigma$ \\
\hline Satellite colour & -0.66 & -6.7 & $2 \times 10^{-11}$ & 0.91 & 0.09 \\
Satellite ${ }^{0.1} M_{r}$ & 0.54 & 5.23 & $1 \times 10^{-7}$ & -20.46 & 0.76 \\
$\log \left(r / \mathrm{R}_{200 \mathrm{~m}}\right)$ & 0.21 & 2.12 & 0.03 & -0.87 & 0.32 \\
Satellite $e$ & 0.20 & 2.1 & 0.04 & 0.43 & 0.26 \\
Cluster $e$ & -2.52 & -25.6 & $2 \times 10^{-143}$ & 0.21 & 0.11 \\
$\Delta \log \left(\right.$ cen. $\left.\mathrm{R}_{\text {eff }}\right)$ & -0.64 & -6.4 & $1 \times 10^{-10}$ & 0.02 & 0.15 \\
cen. ${ }^{0.1} M_{r}$ & 0.58 & 5.01 & $1 \times 10^{-6}$ & -22.35 & 0.49 \\
cen. colour & -0.28 & -2.8 & 0.005 & 0.98 & 0.07 \\
$P_{\text {cen }}$ & -0.46 & -4.2 & $2 \times 10^{-5}$ & 0.87 & 0.17 \\
cen. $e$ & -0.42 & -4.2 & $3 \times 10^{-5}$ & 0.25 & 0.16 \\
$\log$ (richness) & 0.09 & 0.8 & 0.4 & 1.58 & 0.21 \\
\hline
\end{tabular}

\section{THE ORIGIN OF CENTRAL GALAXY ALIGNMENT}

In Section 4.2, we applied linear regression analysis to the nine central galaxy and cluster quantities, and picked the predictors that significantly influence the alignment between central galaxy and its host cluster. We now address the origin of this alignment phenomenon and compare our results with previous studies.

\subsection{Dependence on cluster ellipticity}

Simulations have revealed that clusters are triaxial rather than spherical (Jing \& Suto 2002; Hopkins, Bahcall \& Bode 2005; Kasun \& Evrard 2005; Allgood et al. 2006; Hayashi, Navarro $\&$ Springel 2007), so they look elongated when projected on the sky. The last panel in the second last row of Fig. 9 shows the distribution of projected redMaPPer cluster ellipticities traced by the weighted member galaxy distribution (see Section 2.2.3 for definition of cluster ellipticity), with a mean cluster ellipticity of $\sim 0.20$ and a mean projected semiminor to semimajor axis ratio of $\langle b / a\rangle \sim 0.67$, which agrees with the $N$-body simulation of Hopkins et al. (2005) ( $\langle b / a\rangle \sim 0.67$, at redshift zero), but is rounder than that directly measured through gravitational lensing $(\langle b / a\rangle \sim$ $0.48_{-0.09}^{+0.14}$ in Evans \& Bridle 2009 and $\langle b / a\rangle \sim 0.46 \pm 0.04$ in Oguri et al. 2010).

As shown in Table 3, we find that cluster ellipticity has the most significant influence on the central galaxy alignment signal, with centrals in more elongated clusters having a stronger alignment with the orientation of their host clusters (see also the secondto-last panel in the first row of Fig. 9, which directly displays the correlation between cluster ellipticity and central galaxy alignment). Since the PA for round clusters is not very meaningful, particularly given observational noise, we have examined the correlation trend for clusters with ellipticity $>0.2$, and found the trend that more elongated clusters show stronger alignment still holds.

The influence of cluster ellipticity on central galaxy alignment can be further visualized in the left-hand panel of Fig. 11, where we plot the distribution of the $p_{\text {mem }}$-weighted averaged central galaxy alignment angle for all $p_{\text {mem }}>0.2$ central-satellite pairs in each cluster, $\left\langle\theta_{\mathrm{cen}}\right\rangle_{\mathrm{cl}}=\frac{\sum_{i} p_{\mathrm{mem}, i} \theta_{\mathrm{cen}}}{\sum_{i} p_{\mathrm{mem}, i}}$, against the cluster ellipticity. The sharp boundary on each side is due to the way we define cluster ellipticity. Since we calculate cluster ellipticity via the satellite galaxy distribution, round clusters (with satellites distributed in an almost circularly symmetric way) thus have $\left\langle\theta_{\text {cen }}\right\rangle_{\mathrm{cl}} \sim 45^{\circ}$. More elongated clusters have more potential for going to lower or higher $\left\langle\theta_{\text {cen }}\right\rangle_{\mathrm{cl}}$ values. At fixed cluster ellipticity, the distribution of $\left\langle\theta_{\mathrm{cen}}\right\rangle_{\mathrm{cl}}$ tends to cluster towards the edges of the minimum and maximum available values. As a demonstration, the right-hand panel of Fig 11 shows the results of simulating two fake clusters with fake member galaxies distributed with elliptical symmetry such that the two clusters would have measured cluster ellipticity of 0.5 (green) and 0.3 (red). We then randomized the PA of the simulated central galaxies, and calculated the corresponding $\left\langle\theta_{\mathrm{cen}}\right\rangle_{\mathrm{cl}}$ value. From the scatter plot and histograms of PA central versus $\left\langle\theta_{\text {cen }}\right\rangle_{\mathrm{cl}}$, it is clear that the relationship between central galaxy PA and $\left\langle\theta_{\text {cen }}\right\rangle_{\mathrm{cl}}$ is non-linear, and that this non-linearity is responsible for the shape of the lefthand panel of Fig. 11. However, with more clusters distributed on the $\left\langle\theta_{\text {cen }}\right\rangle_{\mathrm{cl}}<45^{\circ}$ side across the full cluster ellipticity range shown in the left-hand panel of Fig. 11, centrals do prefer to align with their overall satellite distributions.

There are two mechanisms that may be responsible for the strong dependence of central galaxy alignment on cluster shape: (1) the imprint of infall of matter and galaxies into the cluster preferentially along filaments, and (2) the large-scale tidal gravitational field (either primordial, at the time of central galaxy formation, or tidal torquing over time). First, centrals and their parent clusters are both formed via accreting galaxies along filaments, which imprint preferred directions. As a result of these inflows, we expect central galaxies to be aligned with their clusters, especially for relatively young and small clusters with only one dominant filament, leaving an elongated distribution of galaxies (Knebe et al. 2004; Libeskind et al. 2005, 2015). More massive clusters may have experienced several merger events along filaments in various directions during their assembly history. This more complicated history makes the distribution of galaxies in these clusters more disturbed and randomized, resulting in a rounder shape. Indeed, as shown in the second-to-last row of Fig. 9, there is a weak anticorrelation between cluster ellipticity and richness in our data, with richer clusters having a smaller ellipticity. The subsequent violent merger activities may wash out the memory of the primordial filamentary structure, causing a reduction in the alignment signal (Ragone-Figueroa \& Plionis 2007).

However, over the process of virialization, the distribution of galaxies in clusters would again gradually be stretched out along the direction with the surrounding large-scale tidal field, reaching new equilibrium states with a triaxial morphology. At the same time, central galaxies would also gradually be tidally torqued along the new established direction of tidal field. A more anisotropic distribution of satellites could indicate a more intense tidal fields to torque the centrals. It is unclear how important this instantaneous torquing is; Camelio \& Lombardi (2015) demonstrated that at galaxy scales, it is too weak to account for the observed intrinsic alignments, but it is unclear whether it is definitely subdominant for cluster mass scales.

We emphasize that the above two scenarios (anisotropic infall and tidal torquing) are not mutually exclusive and do not necessarily have some sequence in time. They could both operate at various stages of the cluster and central galaxy evolutionary process. Also, according to the linear alignment model (e.g. Catelan et al. 2001; Hirata \& Seljak 2004), the intrinsic alignment is already set by tidal fields at the time of galaxy formation, and it is not clear how relevant these additional processes that operate later may be. 

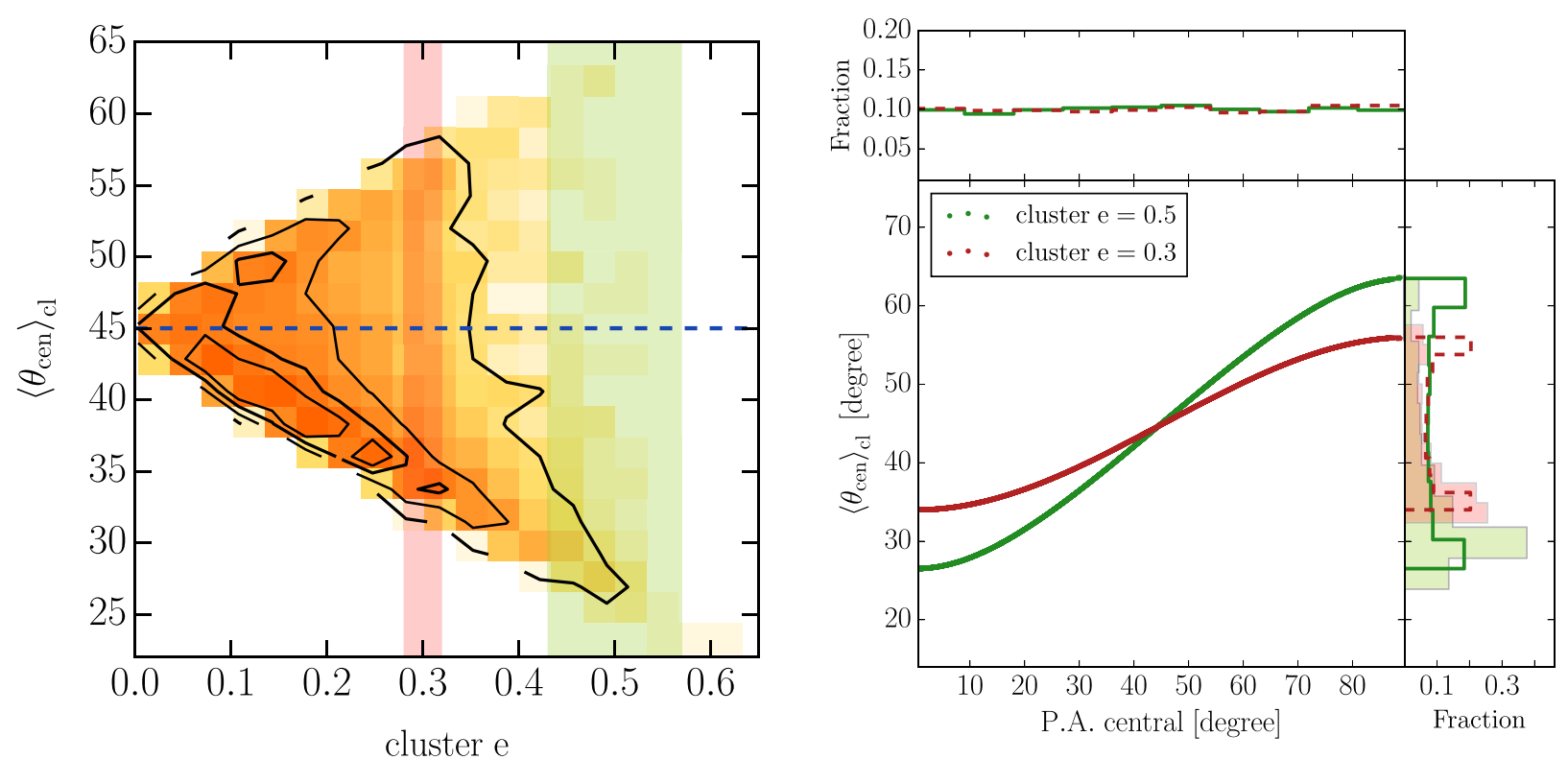

Figure 11. Left-hand panel: contour plot between cluster ellipticity and averaged central galaxy alignment angle for all central-satellite pairs in each cluster, $\left\langle\theta_{\mathrm{cen}}\right\rangle_{\mathrm{cl}}$. The blue dashed line indicates the case $\left\langle\theta_{\mathrm{cen}}\right\rangle_{\mathrm{cl}}=45^{\circ}$, when satellites are randomly distributed within cluster. The light-green (pink) shaded area marks out clusters with ellipticity in the range of $0.43 \sim 0.57(0.28 \sim 0.32)$. For each cluster ellipticity value, there are more clusters distributed in the region below the blue dash line than above, showing the tendency for central galaxy alignments. Right-hand panel: non-linear relationship between central galaxy PA and the derived $\left\langle\theta_{\mathrm{cen}}\right\rangle_{\mathrm{cl}}$ in our simulated data. The simulated clusters with cluster ellipticity of $0.5(0.3)$ are shown in green (red). With completely random distributions of simulated central galaxy PA, the distributions of derived $\left\langle\theta_{\mathrm{cen}}\right\rangle_{\mathrm{cl}}$ tend to peak at their minimum or maximum available values. The light-green and pink shaded histograms are the distributions of $\left\langle\theta_{\mathrm{cen}}\right\rangle_{\mathrm{cl}}$ in our observational data within certain cluster ellipticity ranges as highlighted in the left-hand panel.

\subsection{Dependence on central galaxy effective radius}

As we have shown, the central galaxy effective radius at fixed intrinsic luminosity is also a very significant predictor of the central galaxy alignment effect, with larger sized centrals at a given luminosity exhibiting a stronger degree of central galaxy alignment than smaller sized centrals.

Observations and semi-analytic models have revealed that most massive galaxies grow inside-out, with their extended stellar haloes dominated by accreted stars. The supply of accretion stars may originate from the stellar streams (Belokurov et al. 2006) or the diffuse intracluster light (ICL) which is composed of tidally stripped stars that are gravitationally bound to the cluster potential (Oemler 1976; Lin \& Mohr 2004). These massive accretion-dominated galaxies thus tend to have more extended light profiles compared to galaxies with a stellar component that primarily underwent 'in situ' star formation (van Dokkum et al. 2010; Cooper et al. 2013).

There are two scenarios that can explain the dependence of central galaxy alignment on central galaxy size. First, centrals with more extended morphology may respond more strongly to tidal forces (either the primordial or instantaneous tidal field). Defined as the difference between the gravitational forces at two different positions on an object, the strength of the tidal force would be stronger for objects that have a larger spatial extent. The alternative explanation stems from the closely linked formation and evolution histories of centrals with their host clusters and the surrounding large-scale structures (Conroy, Wechsler \& Kravtsov 2007). As reported in Zhao, Aragón-Salamanca \& Conselice (2015), centrals with extended cD envelopes tend to have larger $R_{\mathrm{e}}$, and are believed to be dominated by baryons from accretion. If there is an abundant supply of accretion stars in some direction aligning with the overall distribution of member galaxies, the central galaxy shape would naturally extend towards the preferred direction of accretion, and thus align with the angle of the member galaxy distribution. We are unable to distinguish between these two scenarios.

\subsection{Dependences on central galaxy luminosity, dominance and centring probability}

According to Fig. 9, central galaxy luminosity, dominance and centring probability are mutually highly correlated with each other, and thus are likely caused by similar physical origins. Here we discuss the dependences of central galaxy alignment on these three predictors.

As revealed in Table 3, we found that $\Delta \eta$ depends significantly on central galaxy ${ }^{0.1} M_{r}$ and $P_{\text {cen }}$, with centrals that are more luminous and have a higher centring probability tending to be more aligned with the cluster PA. Central galaxy dominance, however, was not selected as a featured predictor. This does not mean that central galaxy dominance is not important, but rather that its effect on central galaxy alignment may have been soaked up by the effects of central ${ }^{0.1} M_{r}$ and $P_{\text {cen }}$, so that knowing the central galaxy dominance provides no further help when predicting $\Delta \eta$ if the other two predictors are also known.

Our result is consistent with that of Hao et al. (2011), who also detected a strong dependence of BCG alignment on BCG luminosity based on a sample of richness $\geq 15$ clusters taken from GM$\mathrm{BCG}$, a cluster catalogue constructed based on the red-sequence method (Hao et al. 2010). Also, due to the tight correlation between central galaxy ${ }^{0.1} M_{r}$ and dominance, with more luminous centrals showing higher degree of central galaxy dominance, our result implies that clusters with more dominant centrals should have stronger central galaxy alignment. This agrees with the result of 


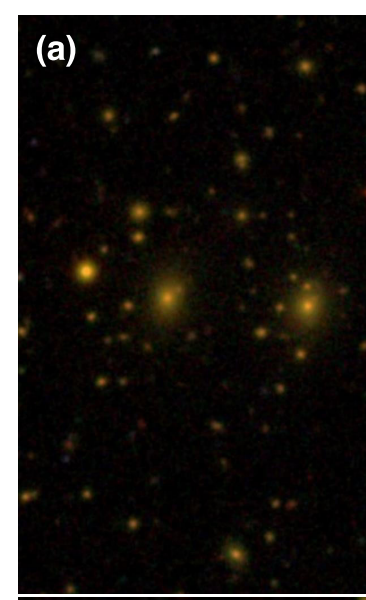

Cen. ${ }^{0.1} \mathrm{Mr}=-23.38$

Cen. ${ }^{0.1} \mathrm{Mr}=-23.38$
Cen. dom $=-0.51$

(c)

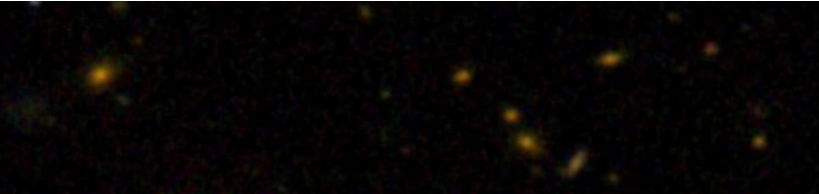

Cen. ${ }^{0.1} \mathrm{Mr}=-23.62$

Pcen $=0.89$

$\therefore \Delta \eta=19.7$

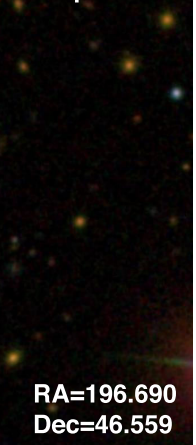

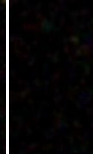

Pcen $=0.97$

$\Delta \eta=36.0$
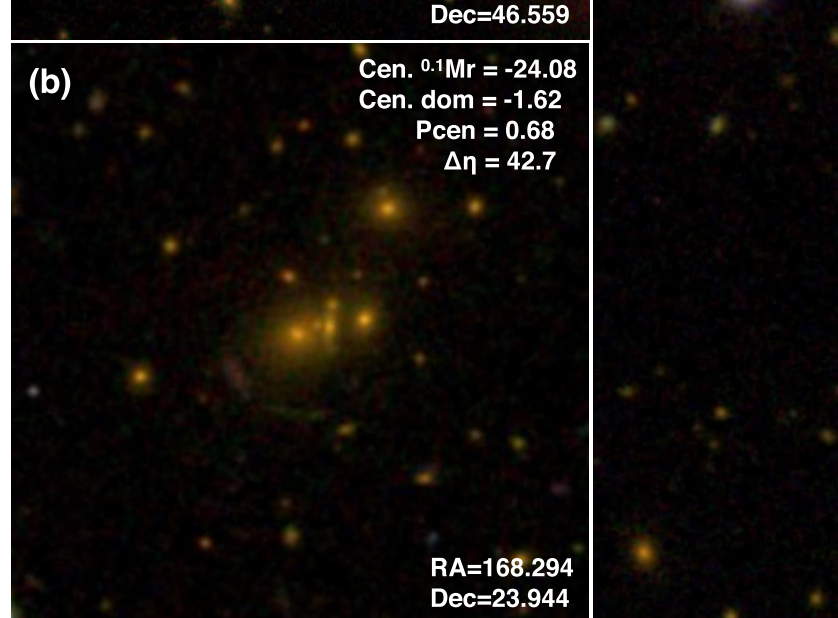

Figure 12. Examples of clusters with central galaxies that are luminous but not dominant. The widths of panels (a), (b), (c) are set to be $\frac{1}{4}, \frac{1}{5}$ and $\frac{1}{2} R_{200 \mathrm{~m}}$, (respectively) of their host clusters.

Niederste-Ostholt et al. (2010). They found that BCG dominant clusters exhibit stronger BCG alignments than less BCG dominant clusters do, with a difference significant at the $4.4 \sigma$ level, based on both the maxBCG cluster catalogue (Koester et al. 2007) and a matched filter cluster catalogue of Dong et al. (2008).

The dependences of the central galaxy alignment signal on central ${ }^{0.1} M_{r}$, dominance and $P_{\text {cen }}$ have their common origin in the following aspects. (1) It may originate from the purity of measurement. Luminous and dominant centrals have a higher probability of sitting closer to the true centre of their DM potential wells (Wen \& Han 2013). This kind of system suffers less contamination from wrong detections, and could therefore end up showing a higher central galaxy alignment signal. (2) Clusters with luminous and dominant centrals are typically more relaxed. More relaxed systems have experienced the uninterrupted (by mergers) influence of surrounding large-scale tidal fields for a longer period of time, and thus it may be more likely for their centrals to align.

Given that central ${ }^{0.1} M_{r}$ and dominance are highly correlated at $\sim 0.6$, it is natural to ask what causes us to select central ${ }^{0.1} M_{r}$ rather than dominance as a featured predictor? To address this question, in Fig. 12, we show some example clusters with luminous but less dominant centrals. As shown, these clusters typically have several bright galaxies, and may still be undergoing significant merging and disruptive interactions. Figs 12(a) and (c) show examples of clusters with their dominantly bright members still some distance away from the centrals. These systems may be not relaxed, but if the centrals' high luminosities and the distributions of their members stem from the same primary avenue of accretion, high alignment signals can still shown even if the centrals are not dominant. This explains why the importance of central ${ }^{0.1} M_{r}$ stands out from central galaxy dominance.

Fig. 12(b) shows the case where the bright members already sank into the potential well of the cluster and are closely interacting with the central galaxy. In this case, the orientation of the central galaxy may be affected temporarily by these closely interacting galaxies, rather than reflecting the tidal field originating from the large-scale environment. The upper-right corner of each panel in Fig. 12 shows some physical properties of the central galaxy. In the case of Fig. 12(b), with several bright galaxies crowded in the central region of the cluster, the central $P_{\text {cen }}$ tends to be low. This demonstrates that $P_{\text {cen }}$ can still be selected as a featured predictor even after selecting central ${ }^{0.1} M_{r}$, because it indicates whether there are other bright galaxies near the central that may reduce the central galaxy alignment with the large-scale tidal field through dynamical processes. While examining images of individual clusters does not give the full picture, it is a way of supplementing the statistical measure of central galaxy alignment from the linear regression analysis.

\subsection{Dependence on central galaxy colour}

We observed that redMaPPer centrals with redder colour show stronger central galaxy alignments. The enhancement of the central galaxy alignment signal among red hosts has also been observed 

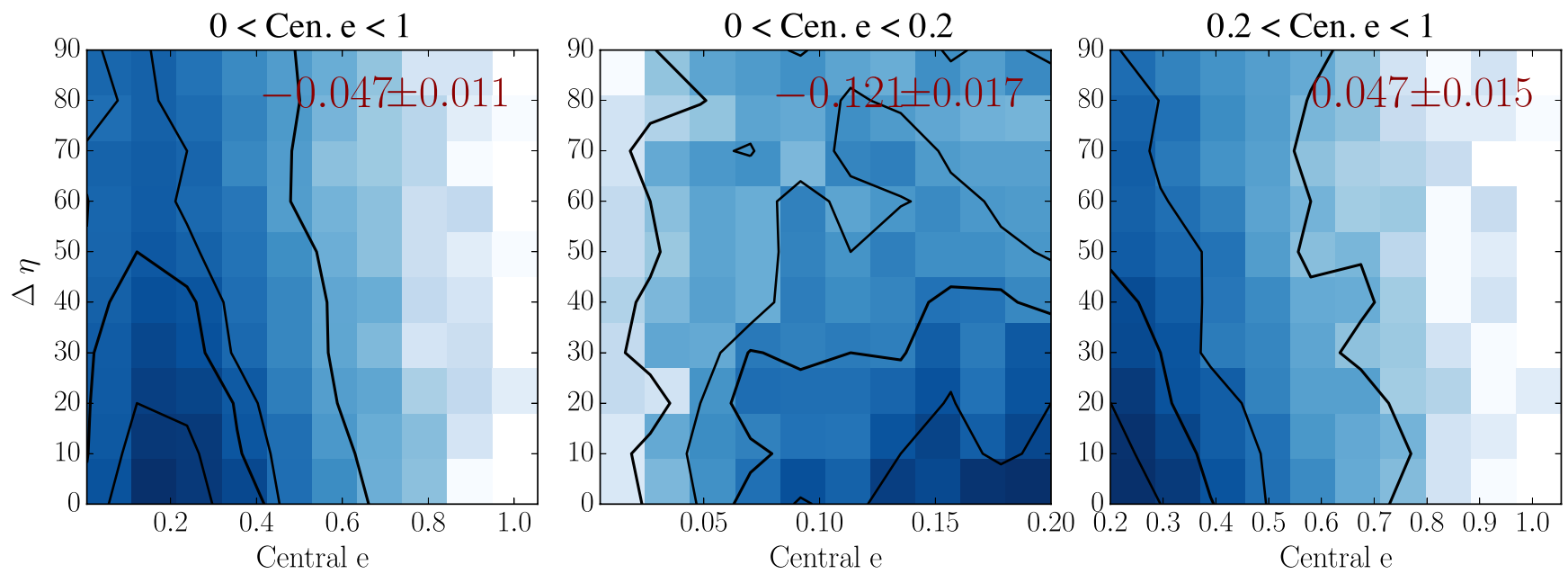

Figure 13. Contours of scatterplots of central galaxy ellipticity versus the PA difference between central galaxy and cluster $(\Delta \eta)$ in different central galaxy ellipticity bins. The left-hand panel shows all of our cluster sample, the middle panel shows only clusters with central galaxy ellipticity below 0.2 , while the right-hand panel plots clusters with their central galaxy ellipticity above 0.2 . The correlation coefficient between $\Delta \eta$ and central galaxy ellipticity is shown at the upper-right corner in each panel.

in systems across a wide range in halo masses. Based on a sample of isolated host galaxies with typically 1-2 satellites, Azzaro et al. (2007) and Agustsson \& Brainerd (2010) found an excess of satellites along the major axis of their centrals only in red-coloured hosts, while satellite distributions are consistent with isotropic around blue hosts. Based on group catalogues spanning from isolated host to cluster scale haloes, Yang et al. (2006), Wang et al. (2008) and Siverd et al. (2009) all found that the alignment signal is only detected in groups with red centrals, and is strongest when considering red centrals and red satellites.

Unlike those previous works, our sample is selected based on the red-sequence method, so the centrals all belong to the red galaxy population. Within the red population, we none the less found that the central galaxy alignment depends on the ${ }^{0.1} \mathrm{Mg}-{ }^{0.1} \mathrm{Mr}$ colour of central galaxy in cluster scale. Galaxy colour indicates the age of the stellar populations. Recent star formation activities induced by the supply of gas from surrounding materials or merger events would cause the central galaxy colour to become less red. Our result thus suggests that central galaxy alignment signal preferentially exists in centrals with relatively old stellar population. For clusters with bluer central galaxies, the alignment of centrals may be disturbed by the recent merger events that also triggered star formation and contributed to the bluer colour.

\subsection{Dependence on central galaxy ellipticity}

Our linear regression shows that central galaxy ellipticity (as defined in equation 2) is negatively correlated with $\Delta \eta$, which means that centrals with larger ellipticity exhibit stronger central galaxy alignment. However, the complication in detecting this trend is that it is more difficult to accurately determine the PAs for round centrals. Statistical scatter in measuring the PAs of more round centrals could in principle drive the effect we have observed, rather than it being a true physical effect. Many studies have required the central galaxy ellipticity to exceed some value in order to avoid this effect, at the expense of introducing some systematic selection effect.

To address this issue, in Fig. 13 we show what happens to the correlation between central galaxy ellipticity and central galaxy alignment angle when we divide the original full sample (left-hand panel) into two ellipticity bins at a value of 0.2 , with 3554 centrals in the $<0.2$ bin, and 4679 centrals in the other. This division reveals that the detected negative correlation of -0.047 in the full cluster sample is dominated by centrals with ellipticity below 0.2 (middle panel), in which a correlation coefficient of -0.12 is measured. These centrals are particularly sensitive to measurement error in the PA, so the observed negative correlation may arise at least in part from measurement error. It may be also possible that this negative correlation originates from real physical mechanisms. The morphology of centrals reflect their formation history. More elliptical centrals may have experienced more anisotropic accretion that contributes to a stronger alignment effect. Distinguishing between measurement error and this real physical effect is difficult.

If focusing on systems with ellipticities above 0.2 (right-hand panel), the central galaxy alignment angle becomes positively correlated at $0.044 \pm 0.015$, meaning that more elongated centrals have smaller alignment signals. We also find that the observed positive correlation is largely driven by the 8 per cent highest ellipticity centrals, with ellipticity $\geq 0.5$. Our result agrees with that of Yang et al. (2006) (see their fig. 2), who found the same tendency using groups with central galaxy ellipticity ${ }^{3} \geq 0.2$.

What causes high-ellipticity centrals to be less aligned? To partially address this question, we visually inspected the images of centrals with very high ellipticities $(\geq 0.6)$ and presented some examples in Fig. 14. Surprisingly, besides the expected cases of highellipticity centrals that are more blue and exhibit discy structures (Fig. 14a) or those with anisotropic ICL (Fig. 14b), we found that in many instances, high-ellipticity centrals are systems with $\geq 2$ bright cores in a single extended envelope (Figs 14c and d). These multiple-core centrals are currently undergoing mergers. During the violent coalescence processes, the PAs of centrals change rapidly and no longer reflect the large-scale matter distribution, resulting in a wide spread in $\Delta \eta$.

We conclude that we should ignore central galaxy ellipticity as an predictor, although it is significantly identified through our variable selection process. The observed negative correlation is mostly

\footnotetext{
${ }^{3}$ The definition of galaxy ellipticity adopted in Yang et al. (2006) is $1-b / a$, based on SDSS isophotal measurement.
} 

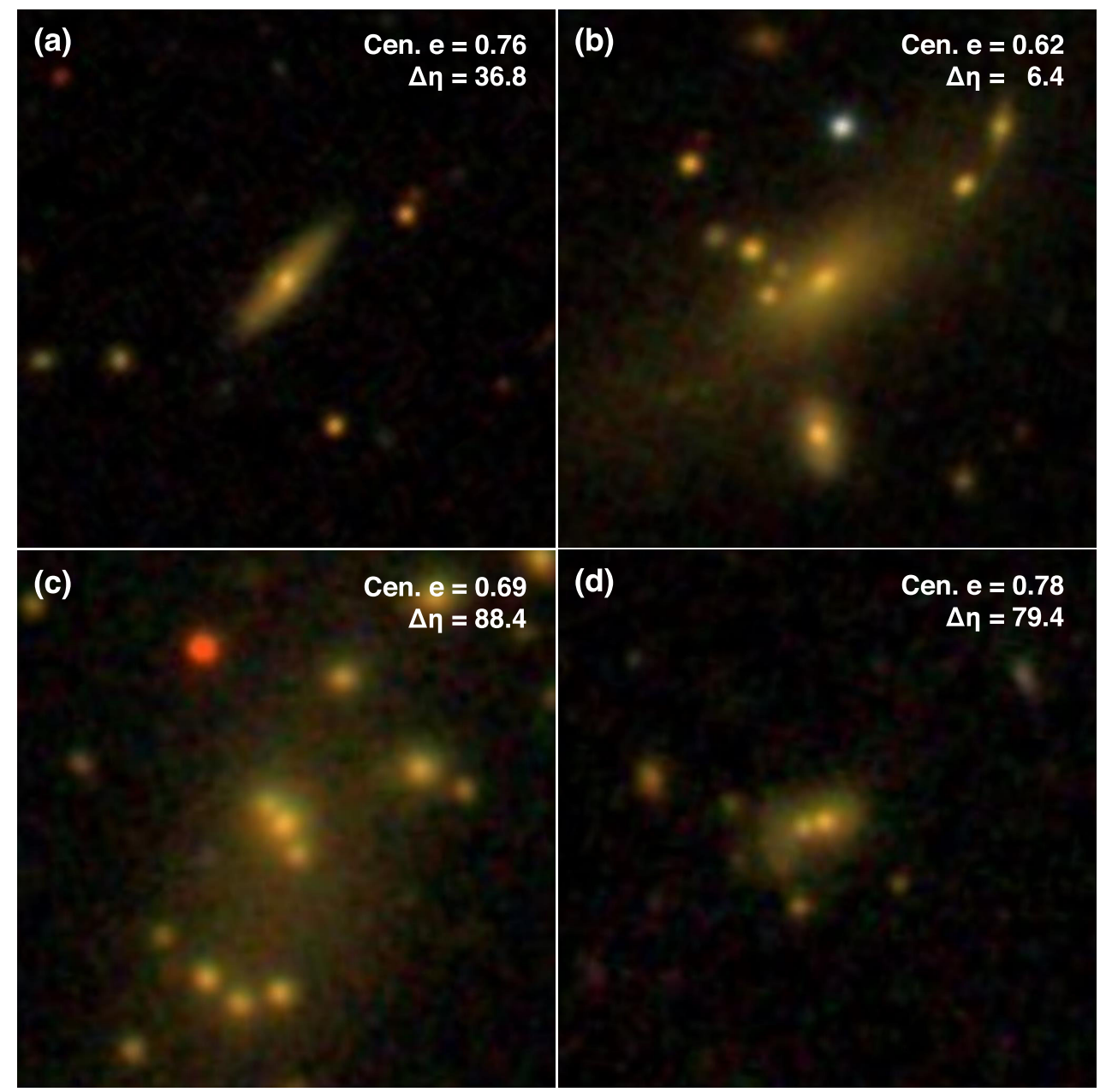

Figure 14. Examples of centrals with measured ellipticity $\geq 0.5$. The central galaxy ellipticity and PA difference between the central galaxy and cluster member galaxy distribution $(\Delta \eta)$ is shown in the upper-right corner of each panel. All panels are $150 \mathrm{kpc}$ on each side. (a) Discy structure central with blueish colour. (b) Central galaxy with elongated ICL. (c) and (d) Centrals with double or more bright cores within common extended envelopes.

driven by rounder centrals whose PA determination is more likely affected by systematics. For more elongated centrals, positive correlation with $\Delta \eta$ is found, and this correlation is possibly driven by centrals at higher ellipticity end. So far we cannot draw a clear conclusion about the impact of central ellipticity on central galaxy alignments. Larger sample size and improved shape measurement method in the future would help us to analyse the non-linear relation between $\Delta \eta$ and central galaxy ellipticity.

\subsection{Dependence on richness}

Richness was selected as a statistically significant predictor when using $\Delta \eta$ as the response variable, but not for the response variable $\theta_{\text {cen. }}$. This result suggests that the impact of richness on the central galaxy alignment signal is marginal. We refer the reader back to the ending of Section 4.3 for related discussion. At similar cluster mass scales, Niederste-Ostholt et al. (2010) also found a slight indication that richer clusters show stronger alignment signals, at $2.3 \sigma$ significance, while Hao et al. (2011) detected no dependence of BCG alignment on richness.

Observationally, richness is a good estimator for the underlying cluster DM halo mass (Rykoff et al. 2012). The weak dependence on richness may be due to the limited range of halo masses covered by the redMaPPer cluster sample. In what follows, we compare papers in which the mean central galaxy alignment angles, $\left\langle\theta_{\text {cen }}\right\rangle$, are provided, and summarize the comparison results in Table 5. Since almost all of the previous works used the isophotal shape measurements, we also turn to our isophotal measurements to fairly compare the $\left\langle\theta_{\text {cen }}\right\rangle$ values. At the mass scale corresponding to galaxy groups, many studies have observed that there is a stronger alignment tendency in richer groups (Yang et al. 2006; Wang et al. 2008; Siverd et al. 2009). As shown in Table 5, the $\left\langle\theta_{\text {cen }}\right\rangle$ value in the highest mass bin of Yang et al. (2006) is consistent with our isophotal $\left\langle\theta_{\text {cen }}\right\rangle$. Going down to even smaller systems, Brainerd (2005) and Agustsson \& Brainerd (2010) have measured the $\left\langle\theta_{\text {cen }}\right\rangle$ using a sample of isolated host centrals. The values of $\left\langle\theta_{\text {cen }}\right\rangle$ are generally larger than that measured in cluster scales. Therefore, we suggest that there truly is some effect of host halo mass on alignments, despite our marginal findings using richness as a mass tracer on cluster mass scales.

Another possible reason that richness may be a less significant predictor is due to the cluster assembly process. Perhaps originally more massive and richer clusters had a stronger primordial alignment with the tidal field, but the subsequent mergers and other major events washed them out, making central galaxy alignments depend only weakly on richness. 
Table 5. Summary of central galaxy alignment measurements. Here we provide a detailed comparison of the observed average central galaxy alignment angle, $\left\langle\theta_{\text {cen }}\right\rangle$, from previous work using a variety of data sets in order to test for the potential evolution of $\left\langle\theta_{\text {cen }}\right\rangle$ with halo mass. Relevant properties of the sample used in these studies are also listed.

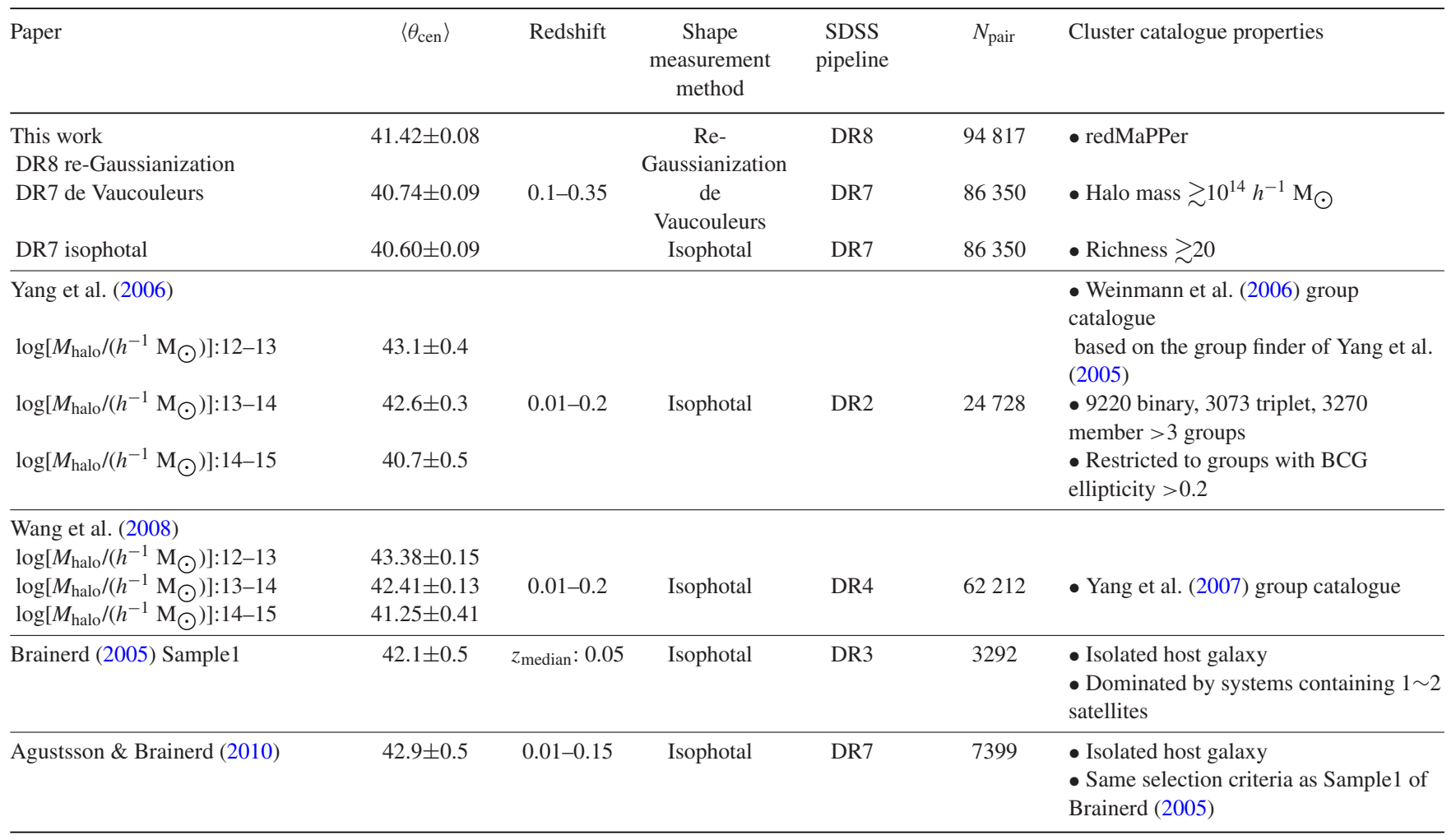

\subsection{Dependence on redshift}

We did not find any significant redshift dependence of central galaxy alignment within the limited redshift range of $0.1-0.35$. In agreement with our observation, Kang et al. (2007) studied the alignment strength from redshift 2 to 0 and found no redshift evolution based on $N$-body simulations with a semi-analytical model for galaxy formation. Based on hydrodynamic simulations, Tenneti et al. (2015b) showed a weak redshift dependence on the intrinsic alignment amplitude at galaxy mass scales, with the alignment signal decreasing at lower redshift. However, based on samples at cluster scale, both Niederste-Ostholt et al. (2010) and Hao et al. (2011) have found that the BCG alignment signal is stronger as redshift decreases within the redshift ranges of $0.08<z<0.44$ and $z<0.4$, respectively. The discrepancies between their results and ours may arise from the following. (1) The two previous studies have considered slightly wider redshift ranges than us such that the redshift-dependent trends become detectable. (2) The observed redshift evolution may be just a reflection of possible combined evolutions with other physical predictors, since those two studies did not consider as many parameters as we do. (3) For studies that based on isophotal shape, there may be more contamination from systematic errors at lower redshift, since for an apparently brighter BCG (at fixed luminosity), its $25 \mathrm{mag} \operatorname{arcsec}^{-2}$ isophote traces a larger radius where the light of BCG is more easily confused with that from other neighbouring satellites. For our redMaPPer sample, when using isophotal shape measurements, we find that the correlation coefficient between $\Delta \eta$ and $z$ is $\sim 1.5$ times higher than that based on re-Gaussianization shape. As discussed in Section 5, this could be partly due to a systematic and partly driven by a real physical effect.
Studying the redshift evolution of the overall central galaxy alignment signal is important for understanding the physical mechanism that is responsible for it. If the central galaxy alignment largely stems from the primordial tidal field at the time of cluster formation (Catelan et al. 2001; Hirata \& Seljak 2004), later merging or virialization processes may weaken the primordial signal (Hopkins et al. 2005). However, if the central galaxy alignment is dominated by signals established from underlying tidal fields acting during the entire lifetime of clusters, or as suggested by Niederste-Ostholt et al. (2010), the primordial alignment signals could be enhanced by the secondary infall episodes, we may expect stronger alignment towards lower redshifts. Currently we lack data to make a convincing conclusion about redshift evolution of central galaxy alignment; further simulations or deeper observational data pushing to higher redshift are needed to further investigate this problem.

\subsection{Dependence on cluster concentration $\Delta_{R}$}

Miyatake et al. (2016) observed that separating redMaPPer clusters with similar richness and redshift distributions into large- $\bar{R}_{\text {mem }}$ and small- $\bar{R}_{\text {mem }}$ populations (see equation 9 for definition of $\bar{R}_{\text {mem }}$ ) yields two cluster subsamples with similar halo masses, but different large-scale biases. Based on the $N$-body simulation in the work of More et al. (2016), $\bar{R}_{\text {mem }}$ is found to be a good indicator for cluster mass accretion rate. Miyatake et al. (2016) thus interpreted the detected difference in large-scale bias as evidence for halo assembly bias, wherein the clustering of haloes depends not only on their mass, but also on other properties related to their assembly histories, such as halo formation time, mass accretion rate, concentration and spin (see e.g. Gao, Springel \& White 2005; Wechsler et al. 2006; Gao \& White 2007; Dalal et al. 2008; Lin et al. 2016). 
Regardless of whether this result indicates assembly bias or some other physical effect can explain the differences in large-scale bias, $\bar{R}_{\text {mem }}$ does correlate with the concentration of the cluster member galaxy distribution, and it is none the less interesting to test whether $\bar{R}_{\text {mem }}$ influences central galaxy alignments. Here we use the parameter $\Delta_{R}$, which removes the richness and redshift dependence of the observed concentration of the member galaxy distribution (equation 10).

We found that $\Delta_{\mathrm{R}}$ has no effect on the central galaxy alignment. In fact, the correlation coefficient between $\Delta \eta$ and $\Delta_{\mathrm{R}}$ is the smallest (0.016) among our predictors, as shown in the upper-right corner of Fig. 9. Moreover, the last row of Fig. 9 shows that $\Delta_{R}$ does not have any $>10 \sigma$ correlations with other parameters, and is therefore relatively independent from the rest of the parameter space considered in this work.

\section{THE ORIGIN OF ANGULAR SEGREGATION OF SATELLITES}

We find that the angular segregation of satellites with respect to their central galaxy major axis direction depends strongly on satellite colour and ${ }^{0.1} M_{r}$, and weakly but still significantly on $\log \left(r / R_{200 \mathrm{~m}}\right)$ and satellite ellipticity, as shown in Table 4 in Section 4.3. In the following we discuss the possible origins of these dependences, and compare our results with previous work. We remind the reader that instead of considering all satellite galaxies, our analysis is only based on red-sequence satellites with membership probability above 0.8 according to the redMaPPer algorithm.

\subsection{Dependence on satellite colour}

The colour of the red-sequence satellites is the strongest predictor of their angular segregation, with redder satellites tending to preferentially lie along the major axis direction of centrals. This result agrees with previous work that considered satellites in a wider colour range and revealed that the distribution of redder satellites shows more anisotropy than that of bluer ones (Yang et al. 2006; Azzaro et al. 2007; Faltenbacher et al. 2007; Wang et al. 2008; Agustsson \& Brainerd 2010).

Part of the dependence on satellite colour may originate from galaxy properties in filaments connected to clusters. Clusters assembled mainly by accreting satellites from surrounding filaments (e.g. Onuora \& Thomas 2000; Lee \& Evrard 2007). As a result, galaxy properties in filaments may leave some imprint on substructures within clusters. Using a filament catalogue (Chen et al. 2016) constructed from SDSS, Chen et al. (2015a) found that red galaxies are on average closer to filaments than blue galaxies. Hence, the observed angular segregation of redder satellites may be due to their being preferentially accreted along filaments, which likely have more tendency to align with the major axes of centrals (see also Kang et al. 2007).

Another possible explanation for the angular segregation by colour is related to environmental quenching. Galaxies in denser environments are redder than galaxies of similar mass in less dense environments (Peng et al. 2010, 2012). Thus, satellites falling along denser filamentary channels would tend to be redder than those falling into the cluster from the field (Martínez, Muriel \& Coenda 2016). Those falling into the cluster along filaments already prequenched. Also, satellites orbiting closer to the major axis direction of centrals should experience higher environmental quenching efficiency due to the higher matter density there.

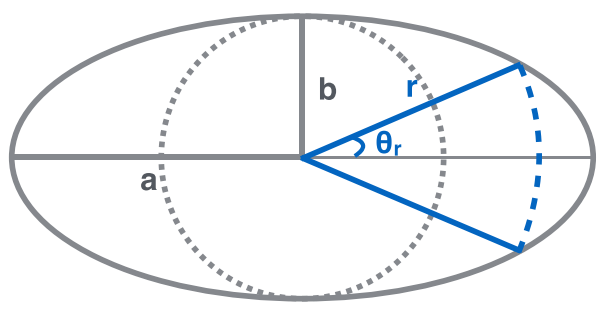

Figure 15. Illustration of how cluster ellipticity can lead to a false detection of dependence of $\theta_{\text {cen }}$ on $\log \left(r / R_{200 \mathrm{~m}}\right)$.

\subsection{Dependence on satellite luminosity}

More luminous satellites are more likely to lie along the major axis directions of centrals. For isolated host-satellite systems, Agustsson \& Brainerd (2010) have also found a consistent trend.

The satellite luminosity dependence may also have its origin from galaxy properties in filaments. By analysing galaxies in filaments, Chen et al. (2015a) found that more massive galaxies tend to be closer to filaments than lower mass galaxies. Li et al. (2013) observed that there is a significant alignment between the orientations of brightest satellite galaxies with the major axes of their groups, suggesting that brightest satellite galaxies entered their host groups more recently than other satellites. Using $N$-body simulations, van den Bosch et al. (2016) have also shown that subhaloes with a larger mass at the time of accretion (a quantity used to link with galaxy stellar mass through abundance matching) tend to be accreted at a later time (see their fig. 5) with smaller orbital energy (i.e. on more bound orbits, see their fig. 9). Combining these previous findings, the physical picture is that more luminous satellites are more likely in-falling from filaments connected to clusters. Since they are accreted by the cluster at a later time, they have not yet orbited enough to lose the imprint of their original large-scale structure. Furthermore, with smaller orbital energy at infall, their dynamics would be more easily influenced by the overall mass distribution in the cluster, and thus as they settle into orbit in the cluster potential well they are more likely to remain along the major axis direction of the central galaxy.

\subsection{Dependence on satellite-central distance}

We found that the satellite-central distance is a statistically significant predictor of the angular segregation of satellites, with those closer to centrals being more likely to be located along the major axis directions of central galaxies. This may seem puzzling given that the second panel in the first row of Fig. 10 shows that the correlation coefficient between $\log \left(r / R_{200 \mathrm{~m}}\right)$ and $\theta_{\text {cen }}$ is consistent with zero within the error bar. Apparently, $\log \left(r / R_{200 \mathrm{~m}}\right)$ is selected as a feature predictor due to some interplay with another predictor. To identify which other predictor is responsible, we removed one predictor at a time in equation (15) to find which one, when removed, caused $\log \left(r / R_{200 \mathrm{~m}}\right)$ to no longer be selected as a feature predictor.

The result of this process was that satellite-central distance was selected due to the presence of cluster ellipticity in the model. The reason why adding cluster ellipticity results in the selection of $\log \left(r / R_{200 \mathrm{~m}}\right)$ is illustrated in Fig. 15. For satellites with projected distances $r<b$ (the semiminor axis of the cluster), the possible values of $\theta_{\text {cen }}$ can vary between $0^{\circ}$ and $90^{\circ}$, while for those with $r>b$, their $\theta_{\text {cen }}$ values are confined within $0^{\circ}$ and $\theta_{r}{ }^{\circ}<90^{\circ}$ due to the boundary of the region contained by the circularized halo radius. Thus, if satellites were randomly distributed within the elliptical footprint of a cluster, we would expect that more elliptical 
clusters exhibit a stronger anticorrelation between $\log \left(r / R_{200 \mathrm{~m}}\right)$ and $\theta_{\text {cen }}$, with larger $\log \left(r / R_{200 \mathrm{~m}}\right)$ showing smaller $\theta_{\text {cen }}$. The fact that that anticorrelation is not observed suggests that galaxies are not randomly distributed within the elliptical footprint of a cluster, but rather are preferentially located on the major axis to a degree that is more significant at smaller values of $\log \left(r / R_{200 \mathrm{~m}}\right)$. Or viewing in the other way, central galaxies tend to point towards nearby satellites, whose distribution reflects local, smaller scale tidal field.

Several previous studies have also investigated the dependence of projected distance on $\theta_{\text {cen }}$. For isolated host scale, Brainerd (2005) found that strength of anisotropy increases with decreasing projected distance, while Azzaro et al. (2007) claimed that the degree of anisotropy is independent of the projected distance and Agustsson \& Brainerd (2010) also reported no distance dependence for red host galaxies. For galaxy group scale, both Yang et al. (2006) and Siverd et al. (2009) detected stronger central galaxy alignment effects at smaller projected distance. Also, in fig. 2 of Faltenbacher et al. (2007), $\theta_{\text {cen }}$ is smaller in the inner part of haloes than in the outer part for red satellites.

The general physical picture regarding angular segregation of satellites is that it is due to large-scale tidal fields, which leads to preferential infall of satellites along the connected filaments. This picture is also reflected in the identification of cluster ellipticity as an predictor described in Section 6.1. If a cluster's smallscale tidal field always followed its large-scale tidal field, then with the presence of predictor cluster ellipticity (reflecting the direction large-scale tidal field), $\log \left(r / R_{200 \mathrm{~m}}\right)$ (reflecting small-scale tidal field) would not be selected out, as all of its effect would be absorbed in cluster ellipticity. During the chaotic assembly process, a cluster's inner tidal field may differ from its large-scale tidal field. The positive correlation between $\theta_{\text {cen }}$ and $\log \left(r / R_{200 \mathrm{~m}}\right)$ found here implies that smaller-scale local tidal field, either newly established or following along the large-scale tidal field, does play some role in torquing the central galaxies to align with satellites located relatively nearby as well.

\subsection{Dependence on satellite ellipticity}

We observed a (marginally) statistically significant dependence of the angular segregation of satellites along the central galaxy major axis on satellite ellipticity, with rounder satellites exhibiting a stronger tendency to lie along the central galaxy major axis direction.

An intuitive way of interpreting the effect of satellite ellipticity is to link it with related galaxy properties. Rounder galaxies have less disc component and an older stellar population, and thus look redder in colour. Also, luminous galaxies tend to be rounder in morphology. Given the relation between satellite ellipticity, colour and luminosity, they may share similar origins, as we have discussed in Sections 7.1 and 7.2. However, notice in Fig. 10 that the correlation coefficients for satellite ellipticity with ${ }^{0.1} M_{r}$ and colour are $\sim 0.2$ and -0.1 , respectively, meaning that there exists other physical origins different from the effects of ${ }^{0.1} M_{r}$ or colour. We must seek other physical mechanisms that are more tightly linked to the satellite ellipticity itself.

One possible mechanism for the preference of rounder satellites to lie along the major axis directions of centrals may be their frequent interaction with nearby galaxies. According to Kuehn \& Ryden (2005), harassment processes due to close encounters with neighbouring galaxies make galaxies rounder. Also, Rodríguez, Padilla \& García Lambas (2016) found that elliptical galaxies in groups, where more disturbing events are likely to happen, are more spherical than field elliptical galaxies with similar intrinsic properties. Therefore, satellites residing near the major axis directions of centrals are more likely to be harassed due to the higher number density there. Besides the effect of shaping galaxies, higher frequency interactions with other members let satellites experience through more phase mixing and relation processes, thus speeding up their sinking on to the plane of central galaxy, as the gravitational potential is deeper there.

\section{SUMMARY AND CONCLUSION}

In this work, we investigate the central galaxy alignment effect using the redMaPPer cluster catalogue. We use three kinds of measurements of the central galaxy PA from the SDSS derived from previous work: re-Gaussianization, de Vaucouleurs and isophotal shapes, compare the derived central galaxy alignment strength among them, and discuss possible systematic effects. To identify the dominant predictors of the central galaxy alignment signal, we include as many potential physical parameters as possible, and apply forwardstepwise linear regression to quantify the statistical significance of these parameters as predictors, as well as to properly account for correlations between them.

Our analysis has two steps. In step one, we regress the PA difference between the central galaxy and cluster shape (as traced by the member galaxy distribution, a proxy for the DM halo shape), $\Delta \eta$, against central galaxy and cluster related quantities. The goal of this step is to identify the central galaxy and cluster properties that most significantly affect their alignment. In step two, we regress the angular location $\theta_{\text {cen }}$ of each member galaxy with reference to its central galaxy major axis direction against several satellite-related quantities, in order to identify important predictors for the angular location of the satellite with respect to the central galaxy major axis. Our key results are as follows.

(i) The detected central galaxy alignment signal is strongest based on isophotal shape, followed by de Vaucouleurs and reGaussianization shape (see the right-hand panels in Figs 6 and 8). This may be caused by the fact that the isophotal shape traces a galaxy's outermost regions, which are more susceptible to the external tidal fields.

(ii) The central galaxy-cluster alignment is strongest for clusters that are more elongated and higher richness, or that have centrals with larger physical size, higher luminosity, redder colour and higher centring probability.

(iii) The tendency of satellites to reside along the central galaxy major axis direction is strongest for satellites with redder colour, higher luminosity, located closer to its central galaxy and with smaller ellipticity.

As shown, we have selected many predictors that have a statistically significant influence on the central galaxy alignment effect. This implies that central galaxy alignment is a complicated phenomenon potentially involved multiple relevant physical processes during galaxy and cluster formation and evolution, such that it cannot be straightforwardly explained by just few dominant factors. We have discussed in great detail the potential physical origins of these selected predictors in Sections 6 and 7. The most relevant factors

\footnotetext{
${ }^{4}$ Although central galaxy ellipticity is found to be a significant predictor as listed in Table 3, we discussed in Section 6.5 that the correlation between central galaxy ellipticity and $\Delta \eta$ is more complicated than a simple linear relation, which requires further investigation in future work.
} 
seem to be that central galaxy alignment may originate from the filamentary accretion processes, but also possibly affected by the tidal field (either the large-scale primordial tidal field, or the newly established small-scale tidal field after the redistribution of satellites). Also, merger events tend to destroy alignment. From this work, we cannot fully disentangle the relative contributions from the above three effects, or rule out contributions from other possible mechanisms that can increase or reduce central galaxy alignment. We expect future investigations either based on observations or simulations to put tighter constraints on possible central galaxy alignment scenarios.

\section{ACKNOWLEDGEMENTS}

We thank Shadab Alam, Tereasa Brainerd, Yun-Hsin Huang, Wentao Luo, Melanie Simet, Sukhdeep Singh and Ying $\mathrm{Zu}$ for useful comments and discussions. This work was supported by the National Science Foundation under Grant No. AST-1313169. YC is supported by William S. Dietrich II Presidential PhD Fellowship Award. EB is partially supported by the US Department of Energy Grant No. DE-SC0007901.

\section{REFERENCES}

Abazajian K. N. et al., 2009, ApJS, 182, 543

Adelman-McCarthy J. K. et al., 2006, ApJS, 162, 38

Agustsson I., Brainerd T. G., 2006, ApJ, 644, L25

Agustsson I., Brainerd T. G., 2010, ApJ, 709, 1321

Aihara H. et al., 2011, ApJS, 195, 26

Akaike H., 1998, Selected Papers of Hirotugu Akaike. Springer, New York, p. 199

Allgood B., Flores R. A., Primack J. R., Kravtsov A. V., Wechsler R. H., Faltenbacher A., Bullock J. S., 2006, MNRAS, 367, 1781

Azzaro M., Patiri S. G., Prada F., Zentner A. R., 2007, MNRAS, 376, L43

Baxter E. J., Rozo E., Jain B., Rykoff E., Wechsler R. H., 2016, MNRAS, 463, 205

Belokurov V. et al., 2006, ApJ, 642, L137

Bernardi M., Meert A., Vikram V., Huertas-Company M., Mei S., Shankar F., Sheth R. K., 2014, MNRAS, 443, 874

Bernstein G. M., Jarvis M., 2002, AJ, 123, 583

Binggeli B., 1982, A\&A, 107, 338

Blanton M. R., Roweis S., 2007, AJ, 133, 734

Blazek J., Vlah Z., Seljak U., 2015, J. Cosmol. Astropart. Phys., 8, 15

Blumenthal G. R., Faber S. M., Primack J. R., Rees M. J., 1984, Nature, 311,517

Brainerd T. G., 2005, ApJ, 628, L101

Bridle S., King L., 2007, New J. Phys., 9, 444

Bruzual G., Charlot S., 2003, MNRAS, 344, 1000

Burnham K. P., Anderson D. R., 2003, Model Selection and Multimodel Inference: A Practical Information-theoretic Approach. Springer, New York

Camelio G., Lombardi M., 2015, A\&A, 575, A113

Catelan P., Kamionkowski M., Blandford R. D., 2001, MNRAS, 320, L7

Chabrier G., 2003, PASP, 115, 763

Chen Y.-C. et al., 2015a, preprint (arXiv:1509.06376)

Chen Y.-C. et al., 2015b, MNRAS, 454, 3341

Chen Y.-C., Ho S., Brinkmann J., Freeman P. E., Genovese C. R., Schneider D. P., Wasserman L., 2016, MNRAS, 461, 3896

Chisari N. E., Mandelbaum R., Strauss M. A., Huff E. M., Bahcall N. A., 2014, MNRAS, 445, 726

Ciotti L., Dutta S. N., 1994, MNRAS, 270, 390

Conroy C., Wechsler R. H., Kravtsov A. V., 2007, ApJ, 668, 826

Cooper A. P., D’Souza R., Kauffmann G., Wang J., Boylan-Kolchin M., Guo Q., Frenk C. S., White S. D. M., 2013, MNRAS, 434, 3348

Dalal N., White M., Bond J. R., Shirokov A., 2008, ApJ, 687, 12 di Tullio G., 1978, A\&A, 62, L17

di Tullio G. A., 1979, A\&AS, 37, 591

Dong F., Pierpaoli E., Gunn J. E., Wechsler R. H., 2008, ApJ, 676, 868

Dubinski J., 1998, ApJ, 502, 141

Eisenstein D. J. et al., 2001, AJ, 122, 2267

Evans A. K. D., Bridle S., 2009, ApJ, 695, 1446

Faltenbacher A., Li C., Mao S., van den Bosch F. C., Yang X., Jing Y. P., Pasquali A., Mo H. J., 2007, ApJ, 662, L71

Faltenbacher A., Jing Y. P., Li C., Mao S., Mo H. J., Pasquali A., van den Bosch F. C., 2008, ApJ, 675, 146

Friedman J., Hastie T., Tibshirani R., 2001, The Elements of Statistical Learning. Vol. 1, Springer, Berlin

Fukugita M., Ichikawa T., Gunn J. E., Doi M., Shimasaku K., Schneider D. P., 1996, AJ, 111, 1748

Gao L., White S. D. M., 2007, MNRAS, 377, L5

Gao L., Springel V., White S. D. M., 2005, MNRAS, 363, L66

Gunn J. E. et al., 1998, AJ, 116, 3040

Hao J. et al., 2010, ApJS, 191, 254

Hao J., Kubo J. M., Feldmann R., Annis J., Johnston D. E., Lin H., McKay T. A., 2011, ApJ, 740, 39

Hayashi E., Navarro J. F., Springel V., 2007, MNRAS, 377, 50

Hirata C., Seljak U., 2003, MNRAS, 343, 459

Hirata C. M., Seljak U., 2004, Phys. Rev. D, 70, 063526

Hirata C. M., Mandelbaum R., Ishak M., Seljak U., Nichol R., Pimbblet K. A., Ross N. P., Wake D., 2007, MNRAS, 381, 1197

Hogg D. W., Finkbeiner D. P., Schlegel D. J., Gunn J. E., 2001, AJ, 122, 2129

Hopkins P. F., Bahcall N. A., Bode P., 2005, ApJ, 618, 1

Hoshino H. et al., 2015, MNRAS, 452, 998

Hung C.-L., Ebeling H., 2012, MNRAS, 421, 3229

Ivezić Ž. et al., 2004, Astron. Nachr., 325, 583

James G., Witten D., Hastie T., Tibshirani R., 2013, An Introduction to Statistical Learning with Applications in R. Springer-Verlag, New York Jing Y. P., Suto Y., 2002, ApJ, 574, 538

Joachimi B., Mandelbaum R., Abdalla F. B., Bridle S. L., 2011, A\&A, 527, A26

Joachimi B. et al., 2015, Space Sci. Rev., 193, 1

Kang X., van den Bosch F. C., Yang X., Mao S., Mo H. J., Li C., Jing Y. P., 2007, MNRAS, 378, 1531

Kasun S. F., Evrard A. E., 2005, ApJ, 629, 781

Kiessling A. et al., 2015, Space Sci. Rev., 193, 67

Kirk D. et al., 2015, Space Sci. Rev., 193, 139

Knebe A., Gill S. P. D., Gibson B. K., Lewis G. F., Ibata R. A., Dopita M. A., 2004, ApJ, 603, 7

Koester B. P. et al., 2007, ApJ, 660, 221

Kormendy J., 1982, in Martinet L., Mayor M., eds, Saas-Fee Advanced Course 12: Morphology and Dynamics of Galaxies. Observatoire de Geneve, Sauverny, Switzerland, p. 113

Kuehn F., Ryden B. S., 2005, ApJ, 634, 1032

Kuhlen M., Diemand J., Madau P., 2007, ApJ, 671, 1135

Lauer T. R. et al., 2005, AJ, 129, 2138

Lee J., Evrard A. E., 2007, ApJ, 657, 30

Li Z., Wang Y., Yang X., Chen X., Xie L., Wang X., 2013, ApJ, 768, 20

Libeskind N. I., Frenk C. S., Cole S., Helly J. C., Jenkins A., Navarro J. F., Power C., 2005, MNRAS, 363, 146

Libeskind N. I., Hoffman Y., Tully R. B., Courtois H. M., Pomarède D., Gottlöber S., Steinmetz M., 2015, MNRAS, 452, 1052

Lin Y.-T., Mohr J. J., 2004, ApJ, 617, 879

Lin Y.-T., Mandelbaum R., Huang Y.-H., Huang H.-J., Dalal N., Diemer B., Jian H.-Y., Kravtsov A., 2016, ApJ, 819, 119

Lupton R. H., Gunn J. E., Ivezić Z., Knapp G. R., Kent S., Yasuda N., 2001, in Harnden F. R., Jr., Primini F. A., Payne H. E., eds, ASP Conf. Ser. Vol. 238, Astronomical Data Analysis Software and Systems X. Astron. Soc. Pac., San Francisco, p. 269

Mallows C. L., 1973, Technometrics, 15, 661

Mandelbaum R. et al., 2005, MNRAS, 361, 1287 (M05)

Mandelbaum R., Hirata C. M., Ishak M., Seljak U., Brinkmann J., 2006, MNRAS, 367, 611 
Martínez H. J., Muriel H., Coenda V., 2016, MNRAS, 455, 127

Miyatake H., More S., Takada M., Spergel D. N., Mandelbaum R., Rykoff E. S., Rozo E., 2016, Phys. Rev. Lett., 116, 041301

More S. et al., 2016, ApJ, 825, 39

Niederste-Ostholt M., Strauss M. A., Dong F., Koester B. P., McKay T. A., 2010, MNRAS, 405, 2023

Oemler A., Jr, 1976, ApJ, 209, 693

Oguri M., Takada M., Okabe N., Smith G. P., 2010, MNRAS, 405, 2215

Okumura T., Jing Y. P., Li C., 2009, ApJ, 694, 214

Onuora L. I., Thomas P. A., 2000, MNRAS, 319, 614

Padmanabhan N. et al., 2008, ApJ, 674, 1217

Peng Y.-j. et al., 2010, ApJ, 721, 193

Peng Y.-j., Lilly S. J., Renzini A., Carollo M., 2012, ApJ, 757, 4

Pereira M. J., Kuhn J. R., 2005, ApJ, 627, L21

Pereira M. J., Bryan G. L., Gill S. P. D., 2008, ApJ, 672, 825

Pier J. R., Munn J. A., Hindsley R. B., Hennessy G. S., Kent S. M., Lupton R. H., Ivezić Ž., 2003, AJ, 125, 1559

Ragone-Figueroa C., Plionis M., 2007, MNRAS, 377, 1785

Reyes R., Mandelbaum R., Gunn J. E., Nakajima R., Seljak U., Hirata C. M., 2012, MNRAS, 425, 2610 (R12)

Richards G. T. et al., 2002, AJ, 123, 2945

Rodríguez S., Padilla N. D., García Lambas D., 2016, MNRAS, 456, 571

Romanowsky A. J., Kochanek C. S., 1998, ApJ, 493, 641

Rong Y., Liu Y., Zhang S.-N., 2016, MNRAS, 455, 2267

Rozo E., Rykoff E. S., 2014, ApJ, 783, 80

Rozo E., Rykoff E. S., Bartlett J. G., Melin J.-B., 2015a, MNRAS, 450, 592

Rozo E., Rykoff E. S., Becker M., Reddick R. M., Wechsler R. H., 2015b, MNRAS, 453, 38

Rykoff E. S. et al., 2012, ApJ, 746, 178

Rykoff E. S. et al., 2014, ApJ, 785, 104

Sastry G. N., 1968, PASP, 80, 252

Schneider M. D. et al., 2013, MNRAS, 433, 2727

Schwarz G., 1978, Ann. Stat., 6, 461

Sifón C., Hoekstra H., Cacciato M., Viola M., Köhlinger F., van der Burg R. F. J., Sand D. J., Graham M. L., 2015, A\&A, 575, A48

Simet M., McClintock T., Mandelbaum R., Rozo E., Rykoff E., Sheldon E., Wechsler R. H., 2016, preprint (arXiv:1603.06953)

Singh S., Mandelbaum R., 2016, MNRAS, 457, 2301

Singh S., Mandelbaum R., More S., 2015, MNRAS, 450, 2195
Siverd R. J., Ryden B. S., Gaudi B. S., 2009, preprint (arXiv:0903.2264)

Skibba R. A., van den Bosch F. C., Yang X., More S., Mo H., Fontanot F., 2011, MNRAS, 410, 417

Smith J. A. et al., 2002, AJ, 123, 2121

Stoughton C. et al., 2002, AJ, 123, 485

Strauss M. A. et al., 2002, AJ, 124, 1810

Tempel E., Guo Q., Kipper R., Libeskind N. I., 2015, MNRAS, 450, 2727

Tenneti A., Singh S., Mandelbaum R., Matteo T. D., Feng Y., Khandai N., 2015a, MNRAS, 453, 469

Tenneti A., Singh S., Mandelbaum R., Matteo T. D., Feng Y., Khandai N., 2015b, MNRAS, 448, 3522

Tucker D. L. et al., 2006, Astron. Nachr., 327, 821

van den Bosch F. C., Weinmann S. M., Yang X., Mo H. J., Li C., Jing Y. P., 2005, MNRAS, 361, 1203

van den Bosch F. C., Jiang F., Campbell D., Behroozi P., 2016, MNRAS, 455,158

van Dokkum P. G. et al., 2010, ApJ, 709, 1018

Wang Y., Yang X., Mo H. J., Li C., van den Bosch F. C., Fan Z., Chen X., 2008, MNRAS, 385, 1511

Wechsler R. H., Zentner A. R., Bullock J. S., Kravtsov A. V., Allgood B., 2006, ApJ, 652, 71

Weinmann S. M., van den Bosch F. C., Yang X., Mo H. J., 2006, MNRAS, 366,2

Weisberg S., 2013, Applied Linear Regression, 4th edn. Wiley, New York

Wen Z. L., Han J. L., 2013, MNRAS, 436, 275

White S. D. M., Rees M. J., 1978, MNRAS, 183, 341

Yang X., Mo H. J., van den Bosch F. C., Jing Y. P., 2005, MNRAS, 356, 1293

Yang X., van den Bosch F. C., Mo H. J., Mao S., Kang X., Weinmann S. M., Guo Y., Jing Y. P., 2006, MNRAS, 369, 1293

Yang X., Mo H. J., van den Bosch F. C., Pasquali A., Li C., Barden M., 2007, ApJ, 671, 153

York D. G. et al., 2000, AJ, 120, 1579

Zhao D., Aragón-Salamanca A., Conselice C. J., 2015, MNRAS, 448, 2530

This paper has been typeset from a $\mathrm{T}_{\mathrm{E}} \mathrm{X} / \mathrm{L} \mathrm{T}_{\mathrm{E}} \mathrm{X}$ file prepared by the author. 